"C 2019 IEEE. Personal use of this material is permitted. Permission from IEEE must be obtained for all other uses, in any current or future media, including reprinting/republishing this material for advertising or promotional purposes, creating new collective works, for resale or redistribution to servers or lists, or reuse of any copyrighted component of this work in other works." 


\title{
Polarization-Reconfigurable Leaky-Wave Antenna with Continuous Beam Scanning Through Broadside
}

\author{
Shu-Lin Chen, Student Member, IEEE, Debabrata K. Karmokar, Member, IEEE, Pei-Yuan Qin, Member, IEEE, \\ Richard W. Ziolkowski, Fellow, IEEE, and Y. Jay Guo, Fellow, IEEE
}

\begin{abstract}
A simple single-layer reconfigurable leaky-wave antenna (LWA) is presented that has polarization agility as well as beam-scanning functionality. This LWA system realizes a scanned beam that can be switched between all of its linear polarization (LP) and circular polarization (CP) states using only one DC biasing source. A slot-loaded SIW-based LWA is first explored to attain $\mathrm{CP}$ performance with continuous beam scanning through broadside. This CP LWA realizes a measured CP performance with a $3 \mathrm{~dB}$ gain variance within $2.75-3.35 \mathrm{GHz}$ for scan angles ranging from $-28.6^{\circ}$ to $+31.5^{\circ}$. A row of shorted stubs is then incorporated into the CP LWA to obtain similar LP performance. Finally, by introducing PIN diodes into this LP LWA configuration to facilitate reconfigurable connections between the main patch and the shorted stubs, the radiated fields can be switched between all of its CP and LP states. Measured results of all three antennas confirm their simulated performance. It is demonstrated that the main beam of the polarization-reconfigurable LWA can be scanned from $-31.5^{\circ}$ to $+17.1^{\circ}$ with gain variations between 9.5 and $12.8 \mathrm{dBic}$ in its CP state, and from $-34.3^{\circ}$ to $+20^{\circ}$ with them between 7.8 and 11.7 dBi in its LP state.
\end{abstract}

Index Terms-Beam scanning, circular polarization (CP), linear polarization (LP), leaky-wave antenna (LWA), polarizationreconfigurable, traveling wave.

\section{INTRODUCTION}

$\mathbf{M}$ ODERN wireless communications rely on antenna systems that deliver high performance multi-functionality. Leaky-wave antennas (LWAs) are popular examples. They have inherent beam-steering features associated with the source frequency. They have simple feed structures and are easy to fabricate [1]-[8]. These LWA features are critical since they reduce the cost and complexity of the antenna systems developed for many aircraft, missile, and satellite applications.

Various LWA beam scanning properties have been reported. When the fundamental mode of a periodic leaky-wave structure is excited, the main beam can be scanned only in the forward quadrant by varying the frequency. Examples include

Manuscript received XXX. This work was supported by the Australian Research Council (ARC) Discovery Project (DP) 160102219 grant and Discovery Early Career Researcher Award (DECRA) DE170101203 grant. (Corresponding author: Debabrata K. Karmokar.)

S.-L. Chen, P.-Y. Qin, R. W. Ziolkowski, and Y. J. Guo are with the Global Big Data Technologies Centre (GBDTC), University of Technology Sydney (UTS), Ultimo, NSW 2007, Australia. E-mail: (Shulin.Chen@uts.edu.au; Pyqin1983@hotmail.com; Richard.Ziolkowski@uts.edu.au; Jay.Guo@uts.edu.au).

D. K. Karmokar is with the School of Engineering, Faculty of Science and Engineering, Macquarie University, Sydney, NSW 2109, Australia. Previously, he was with the Global Big Data Technologies Center (GBDTC), University of Technology Sydney (UTS), Ultimo, NSW 2007, Australia. (email: dkkarmokar@ieee.org) a periodic half-mode microstrip-line LWA [9] and a transverseslot loaded substrate integrated waveguide (SIW) [10]. On the other hand, when the $n=-1$ spatial harmonic of an one-dimensional (1-D) periodic LWA is excited, backwardto-forward beam steering can be realized [11]-[13]. Unfortunately, because they have an inherent stopband, these LWAs cannot steer their main beam through broadside without significant gain loss. Two conditions should be satisfied simultaneously to eliminate the open stopband. First, a continuous transition between the backward and forward phase constant curves is required. Second, the attenuation constant must also have a similar smooth transition, i.e., it should not have any large jumps or zero values in the neighborhood of the broadside radiation region [14]-[16]. A variety of structures have been reported to suppress the open stopband associated with LWAs. For instance, one can close the stopband in a 1-D periodic LWA by using additional radiating elements, impedance matching techniques, groundless spoof plasmon (SP) structures or dielectric image line-based structures [14][20]. One can also elect to employ composite right/left-handed (CRLH) transmission line-based designs [21]-[27].

Note that all of the aforementioned antennas are linearly polarized. Although circular-polarized continuous beam steering through broadside has been reported [28], [29], scanningcapable, polarization-reconfigurable antennas have not. While these multi-functionalities would enhance system capacity and avoid polarization mismatch [30]-[35], it is nevertheless rather challenging to attain polarization reconfiguration in a LWA structure, especially when its main radiator and ground plane are inherently connected with numbers of shorting vias. This is particularly true if PIN diodes are used for the switches since their polarities are difficult to isolate and bias.

To date, very few 1-D LWAs have been reported to target this issue. In [36], a LWA was reported in which its unit cell is constructed with two microstip lines and two CRLH sections. Choosing the current path along one microstrip line and one CRLH section facilitates exciting one linear polarization (LP) state. The other is produced when the other microstrip line and CRLH section pair is selected. Hence, this system achieves two LP states using two independent configurations rather than one augmented with switching devices having realizable biasing circuits. In [37], two CRLH lines were located symmetrically to radiate the orthogonal $\pm 45^{\circ} \mathrm{LP}$ states. Both LP and circular polarization $(\mathrm{CP})$ states were then attained by introducing two different feed networks to separately excite the two CRLH lines with the appropriate amplitudes and phase differences.

In this paper, a polarization-reconfigurable LWA is devel- 


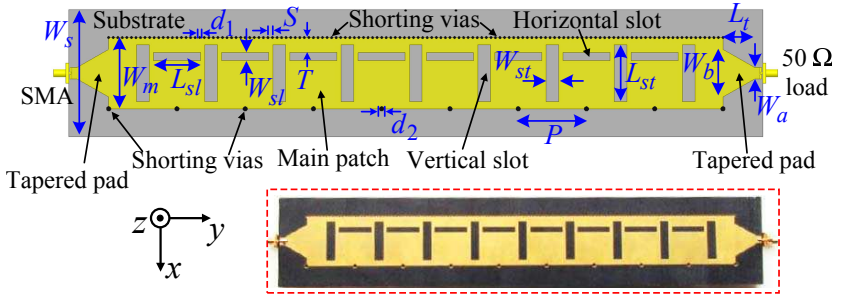

Fig. 1. Top view of the CP LWA. Photograph of the prototype antenna is shown in the bottom inset.

oped with continuous backward to forward beam scanning and with the ability to switch between all of its LP and CP states. To achieve the final design, two variations of nonreconfigurable LWAs, i.e., a CP LWA and a LP LWA, are investigated, fabricated and measured. This work introduces for the first time the realization of polarization reconfigurability with a single feed network. This feature is achieved with a single slot-loaded main patch by introducing PIN diodes that connect the antenna's patch and shorted stubs rather than by combining two radiating elements with different feed networks [37]. Practical DC biasing lines are demonstrated and applied to switch the PIN diodes into their ON and OFF states with only one DC biasing source. In this manner the final reconfigurable LWA achieves switchable polarization states with continuous beam scanning while maintaining a simple feed along with low-profile and easy-integration properties. Its optimized design was fabricated and tested. The measured results of this prototype verify that its main beam can be continuously scanned from $-31.5^{\circ}$ to $+17.1^{\circ}$ with gain variations between 9.5 to $12.8 \mathrm{dBic}$ in its $\mathrm{CP}$ state, and from $-34.3^{\circ}$ to $+20^{\circ}$ with them ranging from 7.8 to $11.7 \mathrm{dBi}$ in its LP state.

The paper is organized as follows. Section II presents the CP LWA with continuous beam scanning through broadside. Section III describes the shorted-stub loaded LP LWA that also exhibits continuous beam scanning. The polarizationreconfigurable, continuous beam scanning LWA is developed in Section IV. The measured and simulated results for each antenna are presented and compared. Conclusions and a complete summary of the performance characteristics of the $\mathrm{CP}$, LP, and polarization-reconfigurable LWAs are given in Section $\mathrm{V}$.

\section{BEAM STEERABLE CP LWA}

A CP LWA with continuous beam scanning through broadside was initially developed. The design, simulation, fabrication, and measurement of this antenna are presented. The measured and simulated performance characteristics are in good agreement.

\section{A. Antenna Configuration}

The CP LWA configuration is shown in Fig. 1. It employs a two-layer copper-clad substrate. The permittivity $\left(\varepsilon_{r}\right)$, loss $\operatorname{tangent}(\tan \delta)$, and height of the substrate were selected to be $3.02,0.001$, and $3.175 \mathrm{~mm}$, respectively. The width of the substrate is $W_{s}$. The main patch is etched on the top layer and centered on the substrate. It has the width $W_{m}$, where
$W_{m}<W_{s}$. The bottom layer is a solid metallic ground plane. The main patch is terminated with two symmetrical, centrally printed tapered matching pads, one at each end. The tapering of each end pad occurs over the length $L_{t}$. Their widths vary from $W_{b}$ (the wide width, connected to the main patch and with $W_{b}<W_{m}$ ) to $W_{a}$ (the narrow width connected to the terminations). These tapered structures are a simple and effective method to attain a wide band impedance matching.

The LWA is fed from the left side with a SMA and is terminated with a $50 \Omega$ load on the right side. The $50 \Omega$ load is used to absorb any power of the traveling wave remaining in the guiding structure to prevent it from being reflected back towards the SMA. The coaxial SMA's inner conductor has a diameter of $1.28 \mathrm{~mm}$. It is connected directly to the narrow width of the left tapered pad. Its outer conductor has the diameter $4.1 \mathrm{~mm}$ and is connected directly to the ground plane.

A row of closely spaced vias with a center-to-center distance $S$ short the upper edge of the main patch to the bottom ground plane. The diameter of these vias is $d_{1}$, where $2 d_{1}>S$. A second row of vias short the lower edge of the main patch to the bottom ground plane. They are separated by a much larger center-to-center distance $P$ and have the diameter $d_{2}$. These two rows of vias form a substrate-integrated-waveguide (SIW) structure.

Two groups of slots, i.e., a set of vertical slots and a set of horizontal slots, are etched on the main patch. The width and length of each vertical slot are $W_{s t}$ and $L_{s t}$, respectively. They are $W_{s l}$ and $L_{s l}$, respectively, for each horizontal slot. The vertical slots are centrally etched on the main patch. The horizontal slots are etched at a distance $T$ from the upper row of shorting vias. The partially open edge formed by the bottom row of shorting vias (with large separation) leads to some power leakage into the far field in the horizontal xy-plane. The antenna radiates primarily in the transverse yz-plane.

The slot-loaded, partially open-edge, SIW unit cell of the CP LWA is shown in the inset of Fig. 2. The length and width of this unit cell are $P$ (the period) and $W_{s}$, respectively. The horizontal and vertical slots are clearly displayed. Half of each horizontal slot is shared with the neighboring unit cells. The upper side of the unit cell is completely shorted with the narrowly-spaced vias; the other is shorted with two half-vias, the other halves being shared with the neighboring unit cells. To achieve a radiation efficiency greater than $90 \%$ over the entire operating band of frequencies, a total of at least nine unit cells must be employed in the finite LWA shown in Fig. 1.

\section{B. Working Mechanisms}

Compared to a conventional open-edge SIW based antenna that radiates through its fundamental $n=0$ mode [9], the antenna in Fig. 1 can radiate through its $n=-1$ spatial harmonic. This radiation mechanism is facilitated by the periodic structure formed with both the horizontal slots and vertical slots. Fig. 2 shows the dispersion diagrams of the unit cell for the $n=-1$ and $n=0$ harmonics. They were obtained using the Eigenmode solver in the high-frequency structure simulator (HFSS) [38]. Note that the dispersion diagrams obtained from 


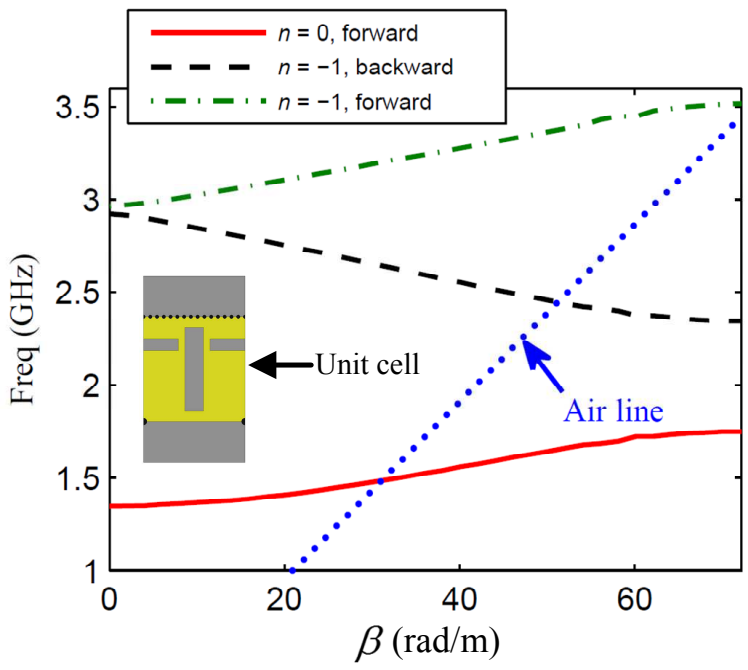

Fig. 2. Dispersion diagrams of the $n=-1$ and 0 spatial harmonics and the unit cell configuration of the CP LWA.

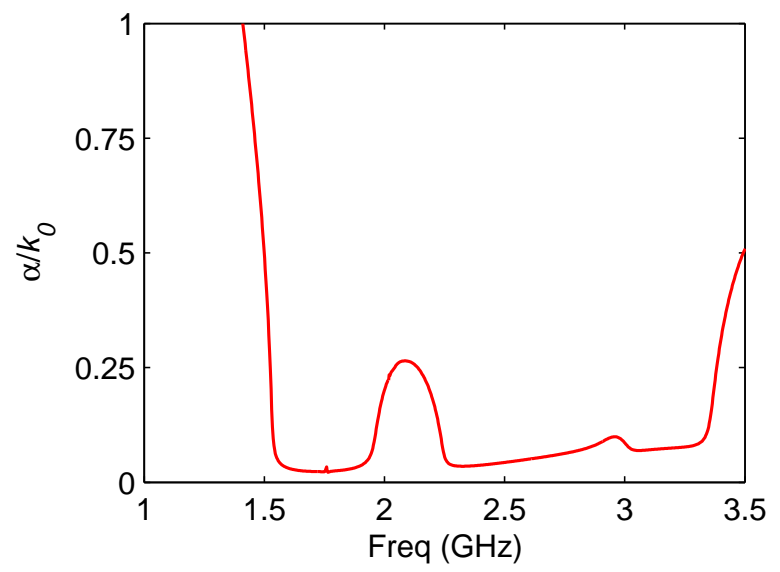

Fig. 3. Normalized attenuation constant of the CP LWA unit cell as a function of the source frequency.

the eigenmode simulation provide a good approximation of the antenna properties. The actual dispersion diagrams can be calculated from the main beam angles obtained from the finite antenna simulations and validated with the corresponding experimental results. It is known that the leaky wave region is on the left side of the air line, and the main beam direction is determined approximately by the following equation [1]:

$$
\theta(f) \approx \sin ^{-1}\left[\frac{\beta_{n}(f)}{k_{0}(f)}\right]
$$

where for a specific frequency, $\theta$ is the main beam direction of the radiation pattern measured from the broadside. The term $k_{0}$ is the wave number in free space, and $\beta_{n}$ is the phase constant of the $n$-th leaky mode, e.g., the $n=-1,0$ modes. The dispersion diagram for the $n=-1$ spatial harmonic indicates that the phase constant achieves a continuous transition between its backward and forward curves. Fig. 3 shows the corresponding normalized attenuation constant $\left(\alpha / k_{0}\right)$ of the CP LWA unit cell as a function of the source frequency. One observes that the attenuation constant has stable values within

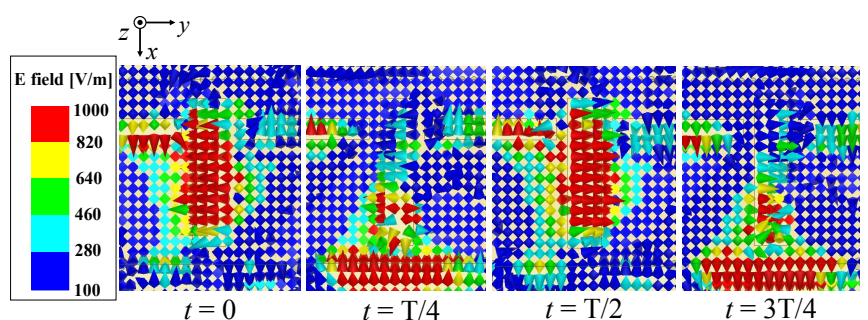

Fig. 4. Top view of E-field distribution on the surface just above one unit cell of the main patch of the optimized CP LWA at $2.8 \mathrm{GHz}$ for four time snapshots separated by a quarter period.

TABLE I

OPTIMIZED VALUES FOR THE CP LWA DESIGN PARAMETERS

\begin{tabular}{|c|c|c|c|c|c|c|c|}
\hline Parameter & $W_{s}$ & $W_{m}$ & $L_{s t}$ & $W_{s t}$ & $L_{s l}$ & $W_{s l}$ & $P$ \\
\hline Value $(\mathrm{mm})$ & 80 & 45 & 36 & 8 & 30 & 5 & 43.5 \\
\hline Parameter & $S$ & $T$ & $d_{1}$ & $d_{2}$ & $L_{t}$ & $W_{a}$ & $W_{b}$ \\
\hline Value $(\mathrm{mm})$ & 2.9 & 9.5 & 1.6 & 3 & 20 & 7.5 & 30 \\
\hline
\end{tabular}

the operational frequency band from 2.5 to $3.35 \mathrm{GHz}$. There is only a small fluctuation around $2.955 \mathrm{GHz}$, i.e., around the transition frequency at which the broadside radiation occurs, which arises from a minor amount of reflection from the unit cell. Furthermore, the attenuation constant values are positive and no zero value is found in the neighborhood of the transition frequency. The behaviors of both the phase and attenuation constants of the $n=-1$ spatial harmonic confirm that the band gap of this CP LWA has been mitigated.

The current CP design was motivated by a previously reported asterisk-shaped slot-loaded partially open-edge SIWbased LWA that accomplished LP beam scanning [39]. The $\mathrm{CP}$ beam scanning feature is realized by optimizing the dimensions of the horizontal and vertical slots that are etched on the main patch with HFSS Optimetrics, i.e., the HFSS parameter analysis. For a clear illustration of the $\mathrm{CP}$ properties, top views of the electric field just above one unit cell for four time snapshots with a quarter period separation are given in Fig. 4 at $2.8 \mathrm{GHz}$. It is seen that the tangential electrical field at $t=0$ is distributed mainly across the vertical slot, and is along the $-y$ direction. Analogously, at $t=\mathrm{T} / 4$, the tangential electrical field is mainly distributed normal to the open-edge of the patch along the $-x$ direction. The E-field distributions at $t=\mathrm{T} / 2$ and $t=3 \mathrm{~T} / 4$ are along the $+y$ and $+x$ directions, respectively. It is clear that the tangential electrical field exhibits a sequential delay in a clockwise direction in one period. Consequently, the antenna radiates a left-handed circular polarization (LHCP) beam. On the other hand, note that when the antenna is fed from the right side and terminated on the left side, a right-handed circular polarization (RHCP) beam is radiated.

\section{Simulated and Measured Results}

The optimized design parameters of the CP LWA are listed in Table I. This design was fabricated, and the resulting prototype is shown in the bottom inset of Fig. 1. The measured and simulated S-parameters are compared in Fig. 5. The simulated $\left|S_{11}\right| \leq-10 \mathrm{~dB}$ frequency bandwidth ranges from 


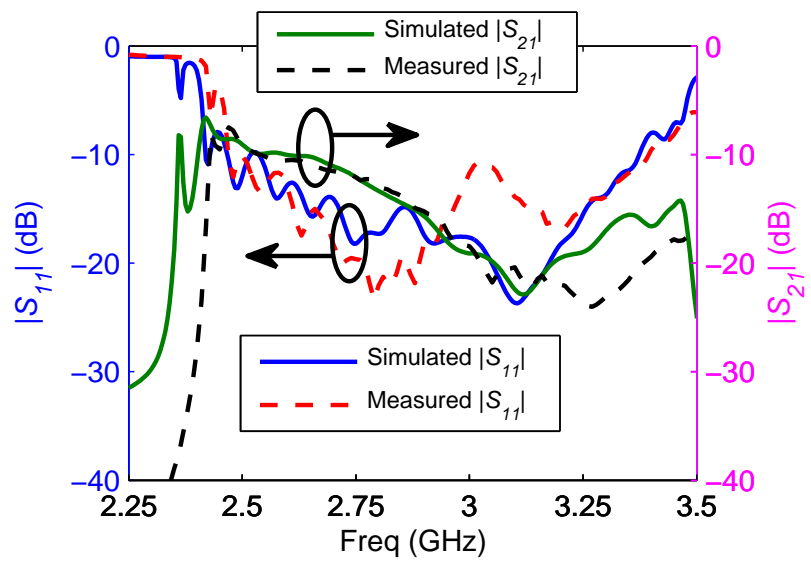

Fig. 5. Measured and simulated S-parameters of the optimized CP LWA.

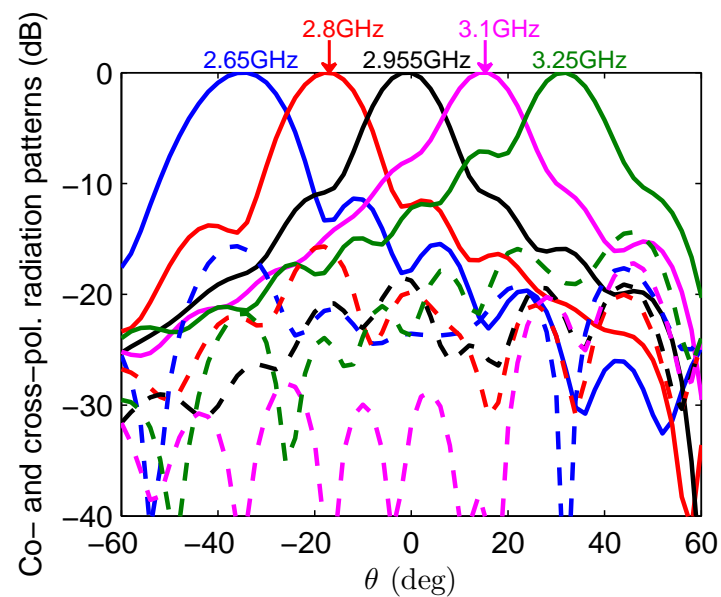

Fig. 6. Simulated radiation patterns of the CP LWA at five frequencies within its bandwidth. Solid line: Co-polarization (LHCP); Dash line: Crosspolarization (RHCP).

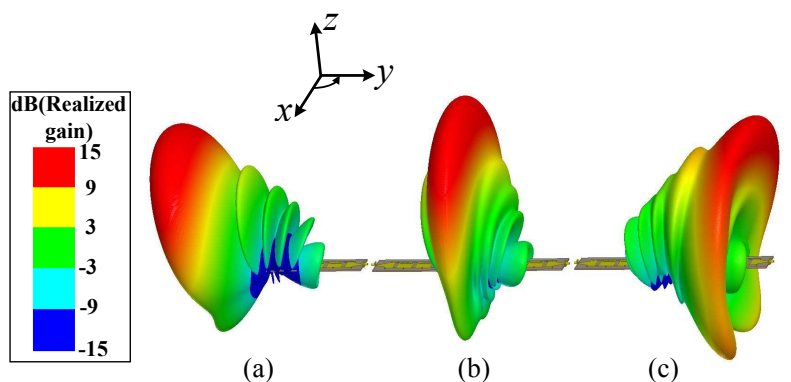

Fig. 7. Simulated 3D realized gain patterns of the CP LWA at three frequencies within its operating bandwidth: (a) 2.65 , (b) 2.955 , and (c) 3.25 GHz.

2.54 to $3.37 \mathrm{GHz}$, while the measured one is from 2.47 to $3.39 \mathrm{GHz}$. The observed peak in the $\left|S_{11}\right|,-10.7 \mathrm{~dB}$, occurs at $3.02 \mathrm{GHz}$, near the measured transition frequency, 3.025 $\mathrm{GHz}$. This feature is associated with the minor fluctuation of the attenuation constant values in that neighborhood. The measured and simulated $\left|S_{21}\right|$ values agree well. Within the measured bandwidth, the measured $\left|S_{21}\right|$ values decrease as the frequency increases, ranging from -7.5 to $-24.0 \mathrm{~dB}$.

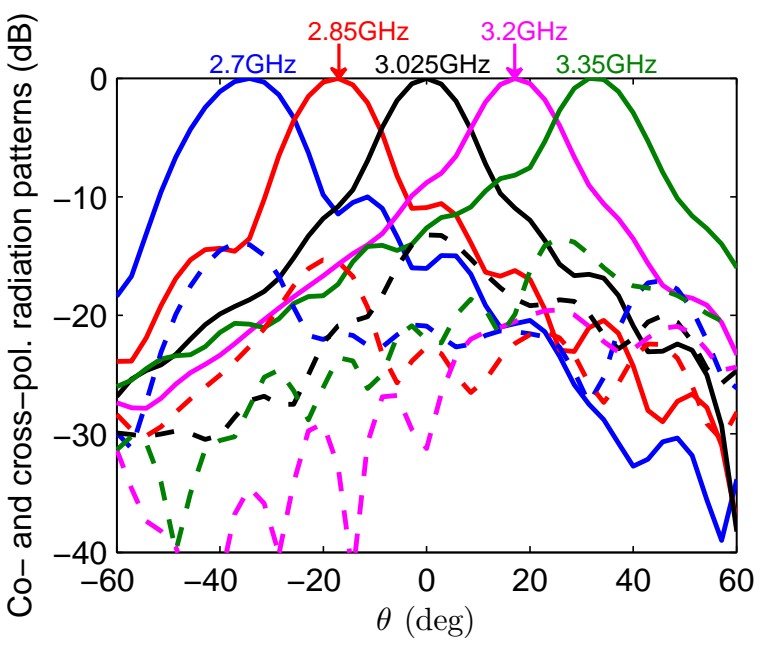

Fig. 8. Measured radiation patterns of the CP LWA at five frequencies within its bandwidth. Solid line: Co-polarization (LHCP); Dash line: Crosspolarization (RHCP).

Fig. 6 shows the simulated co-polarization (LHCP) and cross-polarization (RHCP) radiation patterns at five frequencies within the simulated $-10 \mathrm{~dB}$ impedance bandwidth. As the dispersion diagram in Fig. 2 indicates, the CP LWA radiates a backward beam at lower frequencies. At $2.65 \mathrm{GHz}$ the beam points into the $-34^{\circ}$ direction and then into the $-18^{\circ}$ direction at $2.8 \mathrm{GHz}$. The main beam points at the broadside direction at $2.955 \mathrm{GHz}$. With a further increase in the frequency, the main beam direction moves towards the forward quadrant, e.g., it points into $+14^{\circ}$ and $+32^{\circ}$ at 3.1 and $3.25 \mathrm{GHz}$, respectively. Fig. 7(a), 7(b), and 7(c) show, respectively, the $3 \mathrm{D}$ realized gain patterns at $2.65,2.955$, and $3.25 \mathrm{GHz}$. It is clearly seen that the CP LWA radiates a fan beam at each frequency that has a narrow beamwidth with respect to the yz-plane and a wide beamwidth with respect to the zx-plane. It is found that the simulated main beam angles obtained from the full-wave simulation of complete antenna agree very well with the ones retrieved from the dispersion diagrams in Fig. 2.

The antenna far-field radiation patterns were measured using the Satimo multi-probe spherical near-field system StarLab-18 located at Xiamen University, China. The measured radiation patterns are depicted in Fig. 8 at five frequencies within the measured $-10 \mathrm{~dB}$ impedance bandwidth. The measured LHCP beam points into the $-34.3^{\circ},-17.2^{\circ}, 0^{\circ},+17.2^{\circ}$, and $+31.5^{\circ}$ directions at 2.7, 2.85, 3.025, 3.2, and $3.35 \mathrm{GHz}$, respectively. Note that for a given beam direction the measured frequency is slightly higher than its simulated value. This blue shift is consistent for all of the measured patterns. As a consequence, the frequencies at which the measured patterns are reported were selected to be higher than those simulated in order that the beam directions are nearly the same. In summary, the measured prototype verifies the continuous scanning of the main beam of the CP LWA antenna from the backward to forward directions through broadside.

Fig. 9 shows the simulated and measured realized gain values and the axial ratio (AR) values as functions of the 


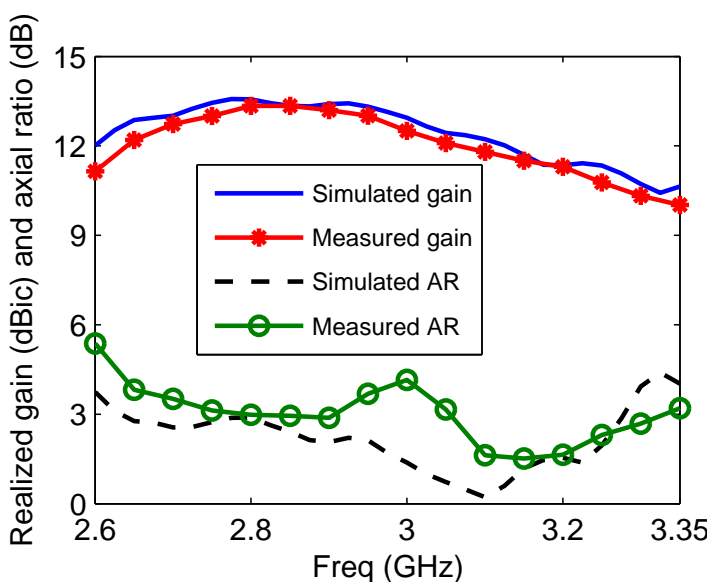

Fig. 9. Measured and simulated realized gain and axial ratio values of the CP LWA as functions of the source frequency.

source frequency. The AR values were obtained at the main beam angle for the corresponding frequency. The simulated $\mathrm{AR} \leq 3 \mathrm{~dB}$ bandwidth is from 2.65 to $3.25 \mathrm{GHz}(600$ $\mathrm{MHz})$, and the realized gain varies between 11.3 and 13.6 $\mathrm{dBic}$ over the same range. The measured frequency band was from 2.75 to $3.35 \mathrm{GHz}(600 \mathrm{MHz})$. The measured realized gain variance is between 10 and $13 \mathrm{dBi}$, i.e., only a $3 \mathrm{~dB}$ gain variance. The measured $\mathrm{AR}$ values are below $3 \mathrm{~dB}$ except when the frequency is around $3.025 \mathrm{GHz}$, the measured transition frequency. The already noted small fluctuation in the attenuation constant values near the transition frequency is responsible for this behavior. However, the associated minor reflections from the unit cell were exacerbated by small fabrication inaccuracies, leading to the increased AR values. The maximum measured AR value was $4.1 \mathrm{~dB}$ at $3.0 \mathrm{GHz}$. Recall that realized gain is proportional to the term $\left(1-\left|S_{11}\right|^{2}\right)$. The differences between the simulated and measured $\left|S_{11}\right|$ values at the transition frequency, $3.025 \mathrm{GHz}$, in Fig. 9 lead to negligible changes in this factor. On the other hand, the fabrication errors introduce differences between the corresponding two orthogonal field components that create the CP fields. This perturbation more significantly impacts their balance and, hence, the AR values near the critical transition frequency.

\section{BEAM STEERABLE LP LWA}

While a CP LWA is a good candidate for the satellite communications, many applications simply require LP performance. It was found that the CP LWA could be modified to obtain just LP fields. A shorted-stub loaded version facilitated the realization of a LP LWA with continuous beam scanning through broadside. The design, simulation, fabrication, and measurement of this antenna are presented. The measured and simulated performance characteristics are in good agreement.

\section{A. Antenna Configuration and Analysis}

The shorted-stub loaded LP LWA unit cell is shown in Fig. 10(a). As illustrated, the shorted stub extends directly from the lower edge of the patch towards the lower side of the

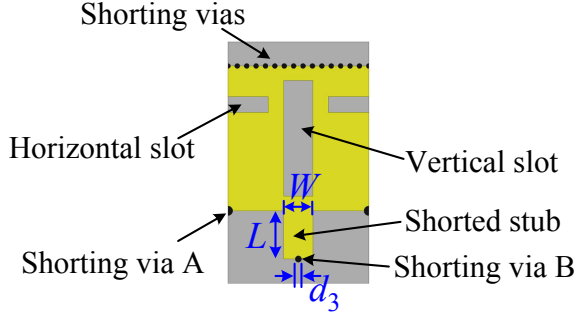

(a)

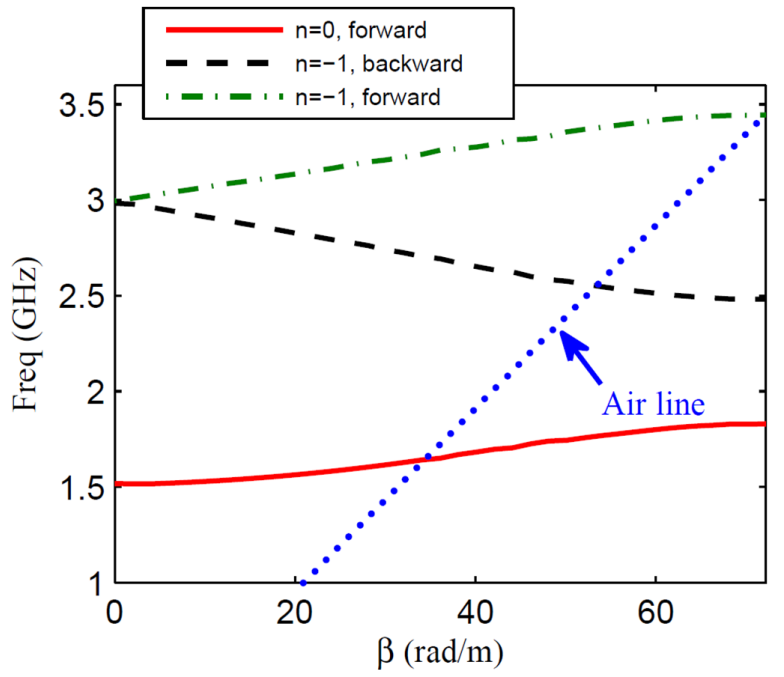

(b)

Fig. 10. LP LWA details. (a) Unit cell configuration. (b) Dispersion diagrams of the $\mathrm{n}=-1$ and 0 spatial harmonics $\left(L=15 \mathrm{~mm}, W=9 \mathrm{~mm}, d_{3}=2\right.$ $\mathrm{mm})$.

substrate. The stub is centered with respect to the vertical slot and is shorted to the ground with the indicated via B. Because of the position of the stub, these additional shorted vias are centered with respect to two of the original shorted vias A. The shorted via $\mathrm{B}$ has a diameter $d_{3}$; it connects the lower end of the stub to the bottom ground plane. The length and width of the shorted stub are $L$ and $W$, respectively. Fig. 10(b) shows the dispersion diagrams of the $n=0$ and $n=-1$ harmonics for the shorted-stub loaded LP LWA. They were obtained using the HFSS Eigenmode solver. It is clear from the intersections with the light line that the shorted-stub loaded LP LWA can realize continuous beam scanning from the backward to forward directions with a continuous transition near 3.0 $\mathrm{GHz}$.

The LP LWA was constructed with nine shorted-stub loaded unit cells as shown in Fig. 11. To explain the LP operating mechanisms of the shorted-stub loaded LP LWA, top views of the electric field just above one unit cell for four time snapshots with a quarter period separation are given in Fig. 12 at $2.8 \mathrm{GHz}$. It is observed that the tangential E-fields are mainly distributed across the vertical and horizontal slots and normal to both edges of the shorted stubs and the bottom edge of the patches. The E-fields on the vertical slots are oriented along the $y$-direction and again lead to strong magnetic currents along the $x$-direction [40], [41]. The tangential E-fields are 


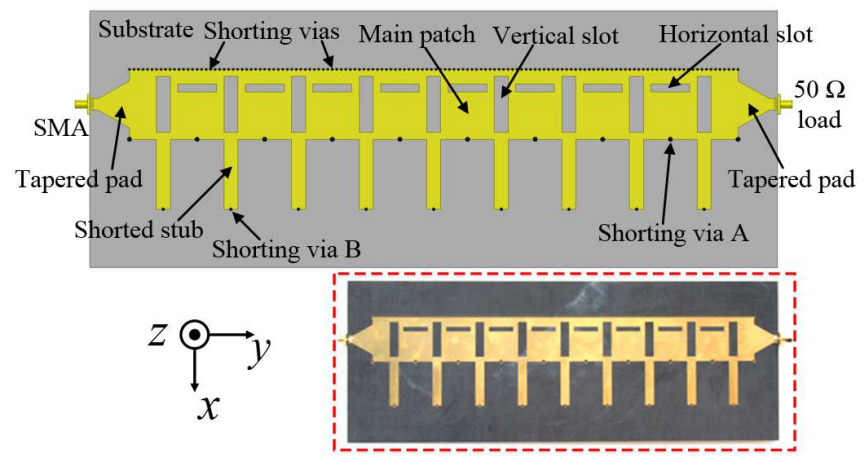

Fig. 11. Top view of the shorted-stub loaded LP LWA. Photograph of the prototype antenna is shown in the bottom inset.

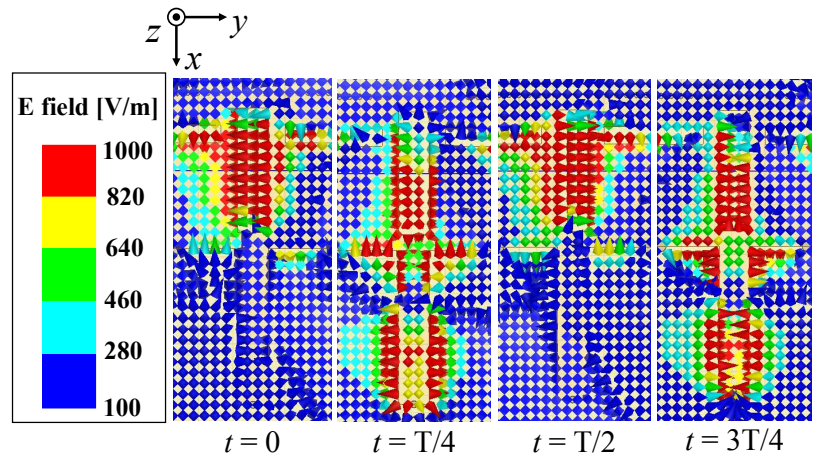

Fig. 12. Top view of E-field distribution on the surface just above one unit cell of the main patch of the optimized LP LWA at $2.8 \mathrm{GHz}$ for four time snapshots separated by a quarter period.

oriented normal to the edges of the shorted stubs introduced in this case, but in opposite directions on opposite sides. Similarly, the electric fields across the horizontal slots are oriented in opposite directions on opposite sides of the vertical ones. Their combination produces two main effects. First, the shorted stubs reduce the strengths of the E-fields along the bottom open-edges of the patches and, thus, the associated $y$ directed magnetic currents. Second, the horizontal slots again provide fine tuning of the overall $y$-directed magnetic currents. By optimizing the dimensions of the shorted stubs and the horizontal slots, their contributions to the far-field cross-pol radiation are reduced significantly. Thus, LP far-fields are realized by the effective magnetic currents oriented along the $x$-directions produced by the vertical slots. The optimized values (in millimeters) for the shorted stubs and the horizontal slots are: $L=45, W=9, d_{3}=2$, and $L_{s l}=25$.

\section{B. Simulated and Measured Results}

The antenna model shown in Fig. 11 was simulated and optimized with HFSS. The optimized design parameter values (in millimeters) are the same as those listed in Table I except: $W_{s}=165, W_{s t}=9$, and $L_{s l}=25$. A prototype of this LP LWA was fabricated. A photo of it is also included at the bottom inset of Fig. 11.

Fig. 13 shows the measured and simulated S-parameters of the optimized LP LWA. The simulated $\left|S_{11}\right| \leq-10 \mathrm{~dB}$ bandwidth is from 2.78 to $3.22 \mathrm{GHz}(440 \mathrm{MHz})$. The corresponding

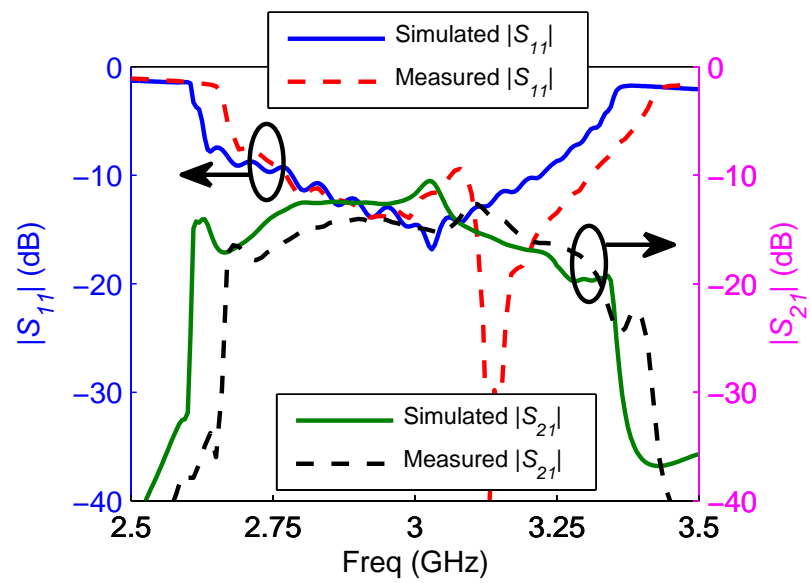

Fig. 13. Measured and simulated S-parameters of the optimized LP LWA.

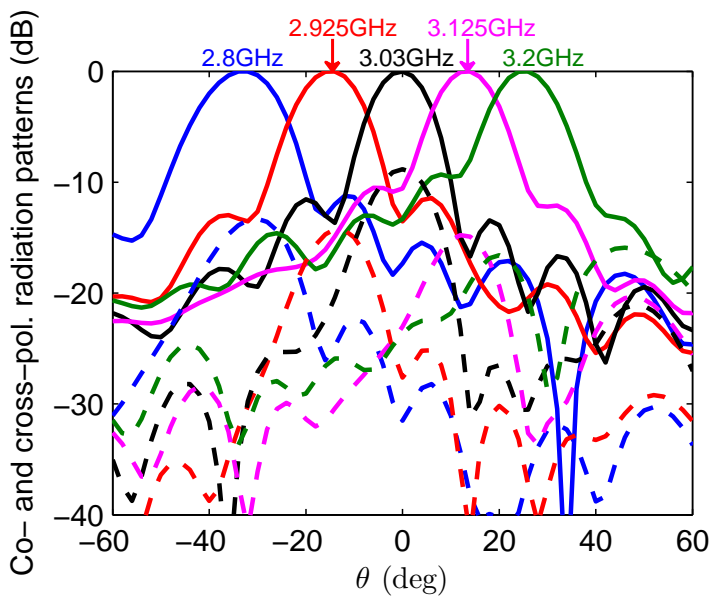

Fig. 14. Simulated radiation patterns of the LP LWA at five frequencies within its bandwidth. Solid line: Co-polarization; Dash line: Cross-polarization.

measured bandwidth is from 2.77 and $3.3 \mathrm{GHz}(530 \mathrm{MHz})$. The peak $\left|S_{11}\right|$ value is $-9.5 \mathrm{~dB}$ at $3.07 \mathrm{GHz}$. The measured $\left|S_{21}\right|$ values are below $-12.6 \mathrm{~dB}$ from 2.77 to $3.3 \mathrm{GHz}$, and they agree well with their simulated values within the $-10 \mathrm{~dB}$ impedance bandwidth.

Fig. 14 shows the simulated co-polarization and crosspolarization radiation patterns for the shorted-stub loaded LP LWA at five frequencies within its impedance bandwidth, and Fig. 15 displays the measured ones. Again, a slight blue shift of the frequency-dependent pattern occurs, i.e, the measured co-polarization main beam points into the $-34.3^{\circ},-14.3^{\circ}$, $0^{\circ},+14.3^{\circ}$, and $+25.7^{\circ}$ directions at $2.85,3.0,3.1,3.2$, and $3.275 \mathrm{GHz}$, respectively. On the other hand, the simulated beam angle is $-32^{\circ},-14^{\circ}, 0^{\circ},+14^{\circ}$ and $+26^{\circ}$ at $2.8,2.925$, $3.03,3.125$, and $3.2 \mathrm{GHz}$, respectively.

Fig. 16 shows the simulated and measured peak realized gain and cross polarization values of the LP LWA as functions of the source frequency. It is clear that the measured peak realized gain curve is similar to the simulated one. The measured peak realized gain increases from $12.7 \mathrm{dBi}$ at 2.85 $\mathrm{GHz}$ to $13.0 \mathrm{dBi}$ at $3 \mathrm{GHz}$, and then decreases to $9.55 \mathrm{dBi}$ at $3.25 \mathrm{GHz}$. It is noted that there is a droop in the measured 


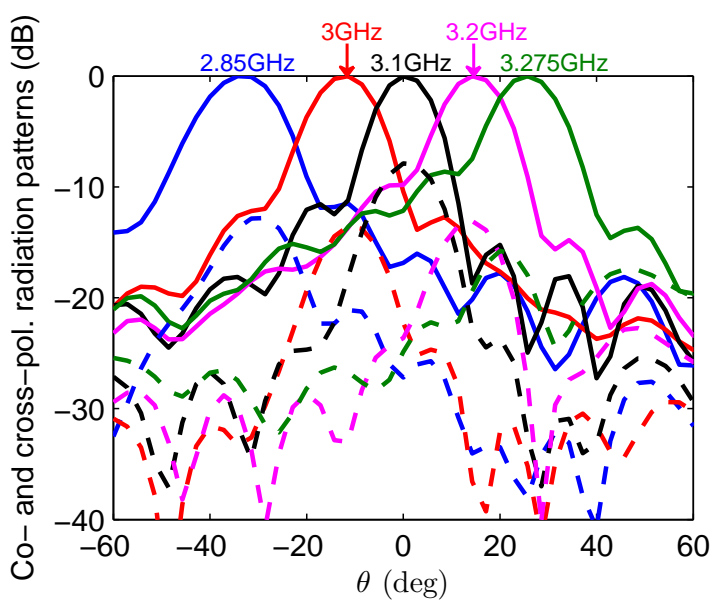

Fig. 15. Measured radiation patterns of the LP LWA at five frequencies within its bandwidth. Solid line: Co-polarization; Dash line: Cross-polarization.

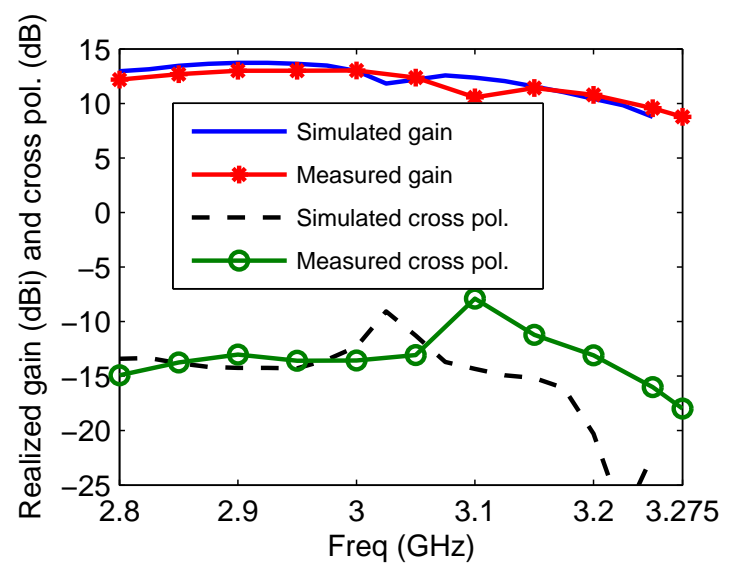

Fig. 16. Measured and simulated realized gain and cross polarization (cross pol.) values of the LP LWA as functions of the source frequency.

value at $3.1 \mathrm{GHz}$, the transition frequency, to $10.6 \mathrm{dBi}$. From 2.85 to $3.275 \mathrm{GHz}$, the measured realized gain variance is $3.45 \mathrm{~dB}$. The measured cross polarization level (obtained at the main beam angle at the corresponding frequency) is stable across the operating frequency band and peaks at $3.1 \mathrm{GHz}$ with the value $-7.9 \mathrm{~dB}$.

\section{Polarization-Reconfigurable Continuous BEAM SCANNING LWA}

To facilitate the development of the desired polarization agile LWA, reconfigurable technology is implemented into the shorted-stub loaded LP LWA structure to switch the polarization between the available CP and LP states. Because the LP LWA was derived from the $\mathrm{CP}$ version by introducing the shorted-stubs, switches are introduced between the original main patch and the shorted stubs to obtain the desired polarization reconfigurability. PIN diodes were selected for those switches; the associated biasing arrangements were developed. The ON-OFF states of the PIN diodes connect or disconnect the shorted stubs to the main patch, yielding the desired selectivity between the LP or CP states.

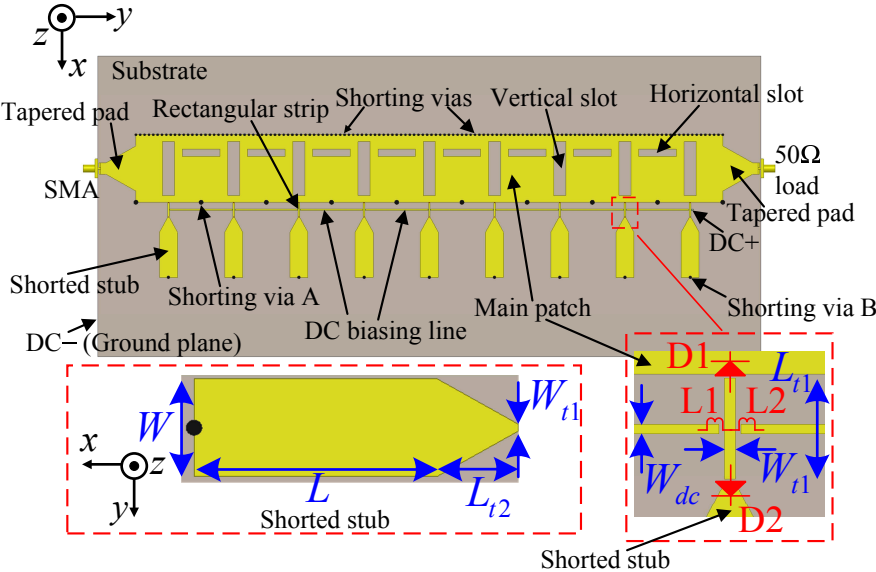

Fig. 17. Top view of the polarization-reconfigurable LWA. The bottom left inset shows the shorted stubs and the right inset shows the diode placements and biasing arrangements.

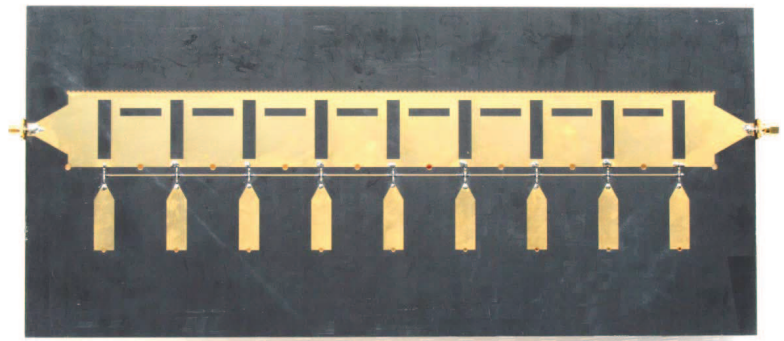

Fig. 18. Photograph of the fabricated polarization-reconfigurable LWA prototype.

The configuration of the polarization-reconfigurable LWA is illustrated in Fig. 17. Details of the modified shorted stub design and the diode placements and biasing arrangements are given in the insets. The stubs were modified to accommodate the presence of the PIN diodes. To connect them to the main patch, the end of the stub nearest to the main patch was tapered to match it to a small rectangular strip whose width matched the much smaller dimensions of the diode. PIN diodes were then introduced to connect the stub to the strip and the strip to the main patch. The PIN-loaded small rectangular strips have the length $L_{t 1}$ and the width $W_{t 1}$. The trapezoidal-shaped taper has its bottom width equal to the shorted stub width $W$ and its top width equal to the strip width $W_{t 1}$. Its height is the length of the taper $L_{t 2}$. When the diodes are switched OFF, the CP LWA configuration is attained. It is noted that if the rectangular-shaped stubs were used, a coupling issue between the rectangular-shaped stubs and the main radiating patch arises that will deteriorate the $\mathrm{CP}$ state of the polarizationreconfigurable antenna. The trapezoidal-shaped taper structure and the small connecting strips between the main patch and the shorted stubs overcome this coupling issue. In particular, the dimensions of the taper structure and the small strips must be carefully optimized to achieve good performance for both the CP and LP states.

As shown in Fig. 17, the two diodes D1 and D2 are aligned in reverse along the $x$-axis, i.e., the positive polarities of the two diodes are soldered to the two ends of the rectangular 


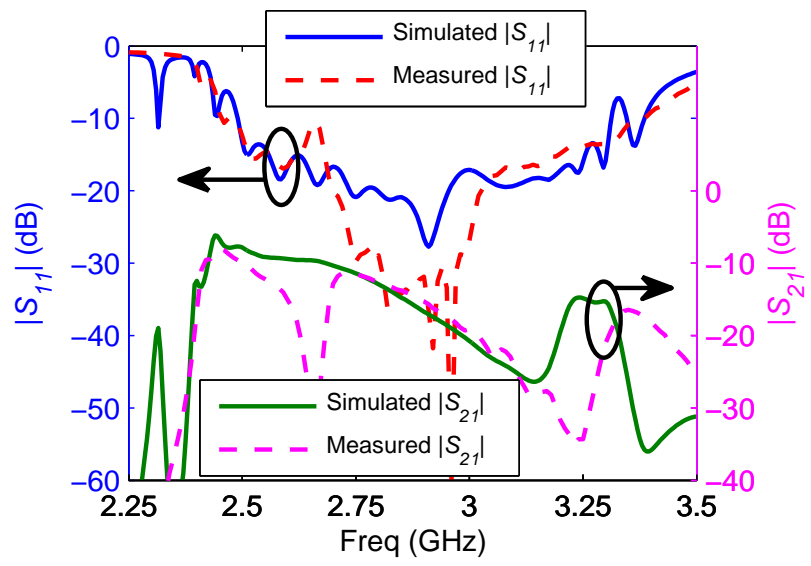

Fig. 19. Measured and simulated S-parameters of the optimized polarizationreconfigurable LWA operating in its CP state.

strip. One negative polarity end is soldered to the main patch; the other one is soldered to a shorted stub and, hence, is connected to the ground plane through its via B. The DC biasing lines are printed along the $y$-axis parallel to the patch edge. They are oriented orthogonal to and are centered in the middle between two of the thin rectangular strips. One can prevent the RF signals from coupling to the DC bias lines and still maintain DC continuity with various approaches. For example, one could utilize meander lines, i.e., distributed inductors, between each end of the DC biasing lines and one of the rectangular strips [42]. However, it was found that they significantly deteriorate the radiation performance of our design. On the other hand, a popular and widely used method is to incorporate chip inductors to block the RF signals. These lumped elements introduce insignificant effects on the antenna's radiation performance [43], [44]. As depicted in the bottom right inset of Fig. 17, inductors L1 and L2 were introduced into our design. They connect the adjacent ends of the DC lines present on both sides of each thin rectangular strip to it. Consequently, only one DC source voltage is needed to bias all of the diodes; and, hence, all the diodes can be switched ON and OFF simultaneously. The DC + in the upper half of Fig. 17 represents the positive polarity of the DC voltage. It is applied to the right most bias line through a small pad that is connected directly to it and to the positive pole of the DC source by a wire passing through a small hole drilled through the substrate and the ground plane. The DCis the negative polarity of DC voltage, which is connected directly to the ground plane. Thus, the DC circuit for biasing the diodes incorporated into this polarization-reconfigurable LWA is simple and effective.

The dimensions of the biasing circuits, including the tapered shorted stubs, were optimized to achieve good radiation and matching performance in both the LP and CP states. The parameter values (in millimeters) of the LP LWA design that had to be modified are: $W_{s}=200, W_{s t}=8, L=30$, and $W=12$. The values for the design parameters (in millimeters) of the thin rectangular lines connecting the main patch and the shorted stubs are: $L_{t 1}=9.2, W_{t 1}=1, L_{t 2}=10, W_{d c}=0.8$.

This optimized design was fabricated and the prototype

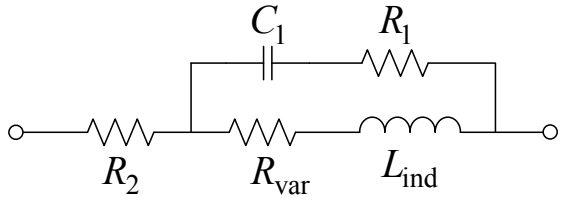

Fig. 20. Equivalent circuit for the Coilcraft 0402HP chip inductors.

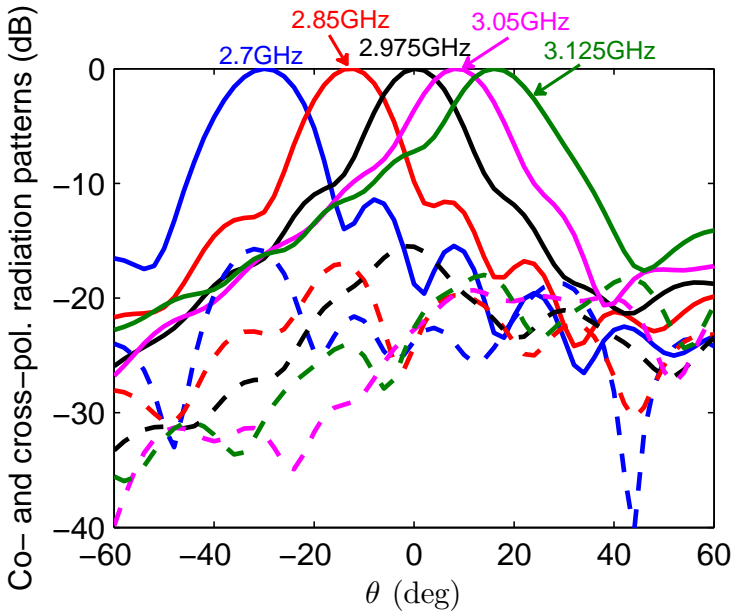

Fig. 21. Simulated radiation patterns of the optimized polarizationreconfigurable LWA at five frequencies within its $\mathrm{CP}$ bandwidth. Solid line: Co-polarization (LHCP); Dash line: Cross-polarization (RHCP).

polarization-reconfigurable LWA is shown in Fig. 15. All of the diodes used for this prototype were the Bar50-02L PIN diodes produced by Infineon Technologies with a 0403 surface mount packaging [45]. According to its datasheet, this diode is equivalent to a resistance of $4 \Omega$ in its $\mathrm{ON}$ state and to a parallel circuit with a capacitance of $0.07 \mathrm{pF}$ and a resistance of $5.0 \mathrm{~K} \Omega$ in its OFF state. Coilcraft chip inductors 0402HP30NX_EU $(30 \mathrm{nH})$ with a self-resonant frequency (SRF) at 2.9 $\mathrm{GHz}$ were also used. It is an important design consideration for any reconfigurable antenna with a biasing network to select inductors with an appropriate SRF. The inductor's SRF should be chosen to be within the desired operating band. According to the datasheet in [46] and confirmed by communications with Coilcraft's technical support, the Coilcraft chip inductors 0402HP-30NX_EU (30 nH) have an SRF of $2.9 \mathrm{GHz}$ and they can block RF signals within the desired operating band, 2.7-3.2 GHz, while maintaining DC continuity. The presence of the inductors can be modeled by their equivalent circuit shown in Fig. 20 in [47], where $R_{1}, R_{2}$, and $R_{\text {var }}$ denote resistors, and $C_{1}$ and $L_{\text {ind }}$ represent a capacitance and inductance, respectively. This circuit introduces a parallel LC resonance within its SRF operating band that yields a very high impedance and, hence, blocks the coupling of the RF currents to the biasing lines [48]. Moreover, a drop in the antenna's gain would occur if the operating frequency was outside the SRF operating band [49]. Full-wave simulations validate that the SRF effects of the inductors have little influence on the antenna's performance. 


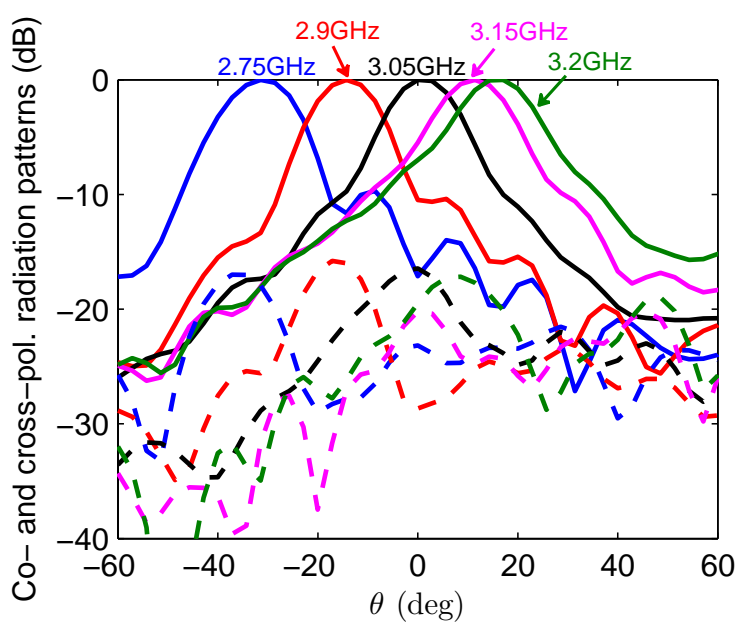

Fig. 22. Measured radiation patterns of the optimized polarizationreconfigurable LWA at five frequencies within its CP bandwidth. Solid line: Co-polarization (LHCP); Dash line: Cross-polarization (RHCP).

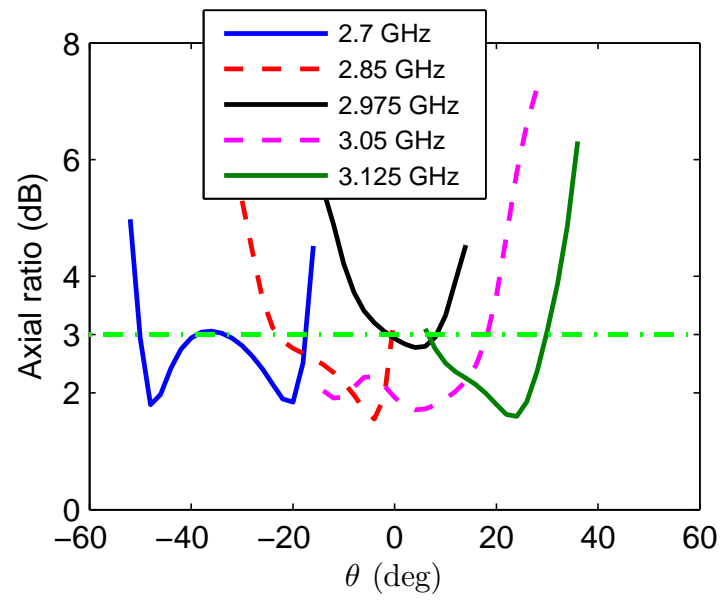

Fig. 23. Simulated AR values as functions of the observation angle at five operating frequencies for the optimized polarization-reconfigurable LWA in its $\mathrm{CP}$ state.

\section{A. Polarization-Reconfigurable LWA Working in Its CP State}

When all of the diodes are switched OFF, i.e., there is no voltage applied between DC + and DC-, the shorted stubs are disconnected from the main patch; and, hence, CP beams are radiated. The measured and simulated $\mathrm{S}$-parameters are shown in Fig. 19. The simulated $\left|S_{11}\right| \leq-10 \mathrm{~dB}$ frequency bandwidth is from 2.5 to $3.3 \mathrm{GHz}(800 \mathrm{MHz})$, while the measured one is from 2.48 to $3.38 \mathrm{GHz}(900 \mathrm{MHz})$. Note that a higher than predicted $\left|S_{11}\right|$ value occurs at $2.66 \mathrm{GHz}$. Additional simulations and measurements have determined that it is caused by either component tolerances or soldering effects associated with the PIN diodes and inductors. Furthermore, the measured $\left|S_{21}\right|$ is below $-9.2 \mathrm{~dB}$ throughout this measured bandwidth.

Figs. 21 and 22 show the simulated and measured radiation patterns of the optimized polarization-reconfigurable LWA at five frequencies within its $\mathrm{CP}$ bandwidth, respectively. The simulated LHCP beam scanning angle is $-30^{\circ},-12^{\circ}, 0^{\circ}$,

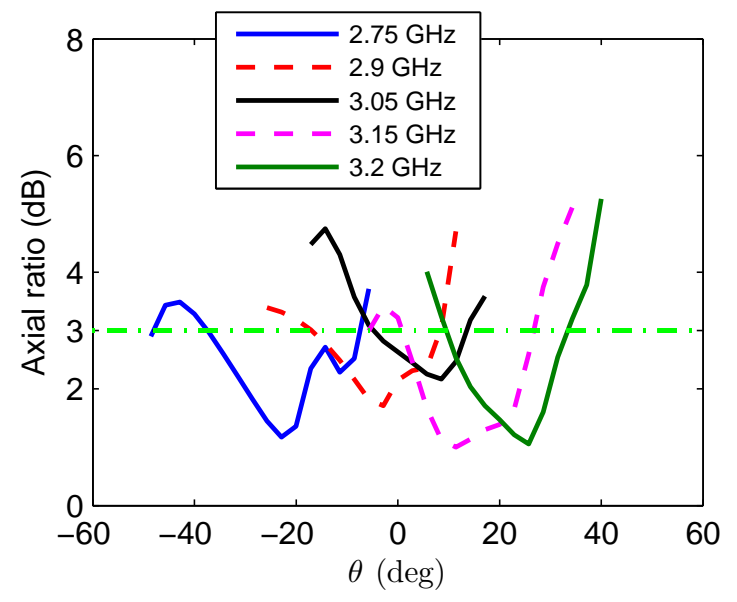

Fig. 24. Measured AR values as functions of the observation angle at five operating frequencies for the optimized polarization-reconfigurable LWA in its CP state.

$+8^{\circ}$, and $+16^{\circ}$ at $2.7,2.85,2.975,3.05$, and $3.125 \mathrm{GHz}$, respectively. The measured result is $-31.5^{\circ},-14.3^{\circ}, 0^{\circ}$, $+11.4^{\circ}$, and $+17.1^{\circ}$ at at $2.75,2.9,3.05,3.15$, and $3.2 \mathrm{GHz}$, respectively. Figs. 23 and 24 show, respectively, the simulated and measured $\mathrm{AR}$ values as functions of the observation angle at five operating frequencies. One finds in Fig. 23 that at the transition frequency $(2.975 \mathrm{GHz})$, the simulated $\mathrm{AR} \leq 3$ beamwidth is from $-2^{\circ}$ to $+8^{\circ}$, i.e., $10^{\circ}$. For other frequencies, it is much wider, i.e., more than $25^{\circ}$. On the other hand, the beamwidths for the measured AR $\leq 3$ values in Fig. 24 are wider than $20^{\circ}$ at those five operating frequencies. Fig. 25 shows the simulated and measured realized gain and AR values as functions of the source frequency from 2.7 to 3.2 $\mathrm{GHz}$. The measured AR values are all below $3.0 \mathrm{~dB}$ within this entire frequency band $(500 \mathrm{MHz})$, while the simulated AR $\leq 3 \mathrm{~dB}$ bandwidth is from 2.7 to $3.13 \mathrm{GHz}(430 \mathrm{MHz})$. The measured AR values are slightly higher than their simulated ones. This difference is again correlated with the fabrication inaccuracies. They lead to small changes in the power radiated by the orthogonal field components and, hence, impact the AR outcomes. The measured gain values are within the range 9.5 (at $3.2 \mathrm{GHz}$ ) to 12.8 (at $2.85 \mathrm{GHz}$ ) dBic. The simulated realized gain values vary between 10.7 and 13.15 dBic over the CP bandwidth. The measured and simulated results are observed to be in reasonable agreement from 2.75 to $3.15 \mathrm{GHz}$ (400 MHz). The simulated total efficiency of the polarizationreconfigurable LWA in its $\mathrm{CP}$ state is shown in Fig. 26. It varies from $75 \%$ and $88 \%$ within the frequency band between 2.7 and $3.2 \mathrm{GHz}$.

\section{B. Polarization-Reconfigurable LWA Working in Its LP State}

When the DC voltage is applied to the bias lines, all of the diodes are switched ON. The polarization-reconfigurable LWA is then in its LP state. Fig. 27 shows the measured and simulated S-parameters versus the source frequency for the polarization-reconfigurable LWA working in its LP state. The measured $\left|S_{11}\right|$ values are below $-10 \mathrm{~dB}$ from 2.7 to $3.3 \mathrm{GHz}$ $(600 \mathrm{MHz})$, except very near to the transition point (i.e., -9.0 


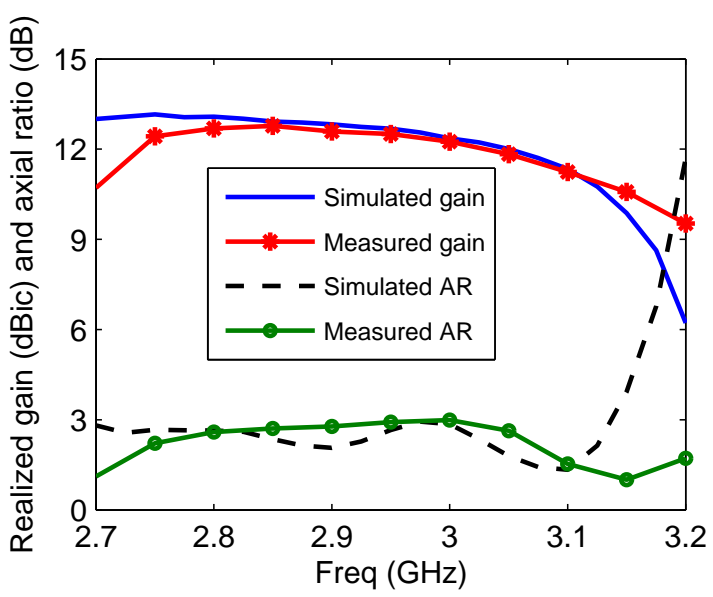

Fig. 25. Measured and simulated realized gain and axial ratio values of the optimized polarization-reconfigurable LWA operating in its CP state as functions of the source frequency.

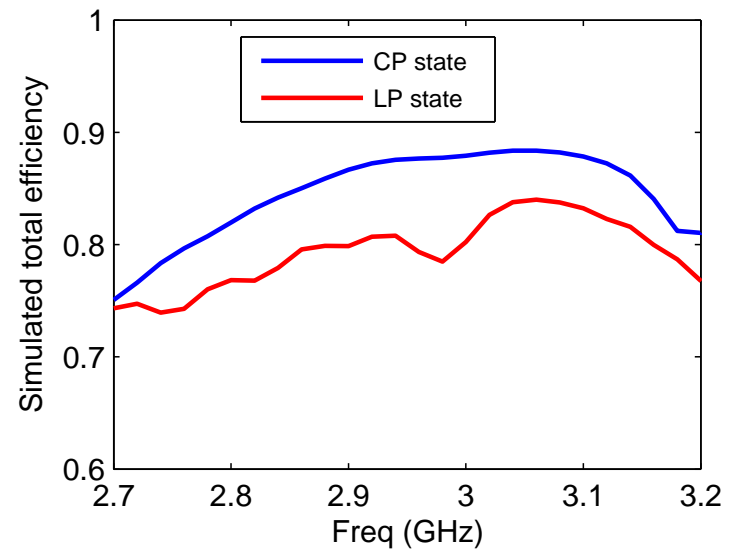

Fig. 26. Simulated total efficiency of the optimized polarizationreconfigurable LWA operating in its CP and LP states.

$\mathrm{dB}$ at $3.05 \mathrm{GHz}$ ). The simulated $\left|S_{11}\right| \leq-10 \mathrm{~dB}$ frequency bandwidth is from 2.56 to $3.24 \mathrm{GHz}(680 \mathrm{MHz})$. Additional simulations and measurements have determined that the higher than predicted $\left|S_{11}\right|$ values around the transition point result are caused by fabrication inaccuracies. The measured and simulated $\left|S_{21}\right|$ values are also shown in Fig. 27. The measured $\left|S_{21}\right|$ values are less than $-14.0 \mathrm{~dB}$ (at $2.75 \mathrm{GHz}$ ) over the entire LP frequency bandwidth. The measured S-parameters agree reasonably well with their simulated values. The simultaneous low $\left|S_{11}\right|$ and $\left|S_{21}\right|$ values indicate good radiation performance.

Fig. 28 shows the simulated co-polarization (LHCP) and cross-polarization (RHCP) patterns of the polarizationreconfigurable LWA for five frequencies within its LP state bandwidth. The simulated LHCP main beam directions are $-36^{\circ},-16^{\circ}, 0^{\circ},+14^{\circ}$, and $+24^{\circ}$ at $2.7,2.85,2.985,3.075$, and $3.15 \mathrm{GHz}$, respectively. Thus, it is verified that the main beam scans from backwards to forwards through broadside with increasing frequency. The measured co-polarization (LHCP) and cross-polarization (RHCP) patterns are shown in Fig. 29. The main beam directions are $-34.3^{\circ},-17.1^{\circ}, 0^{\circ},+14.3^{\circ}$, and

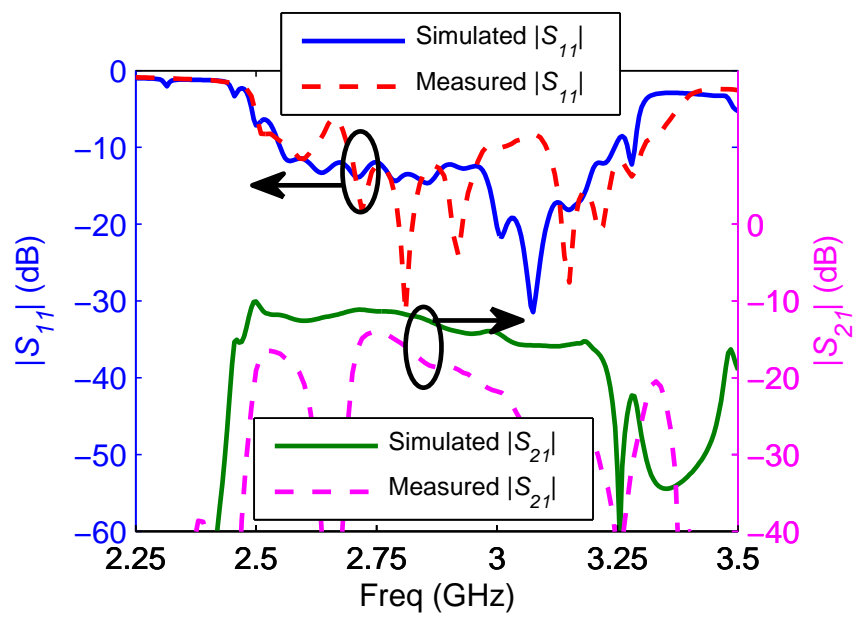

Fig. 27. Measured and simulated S-parameters of the optimized polarizationreconfigurable LWA operating in its LP state.

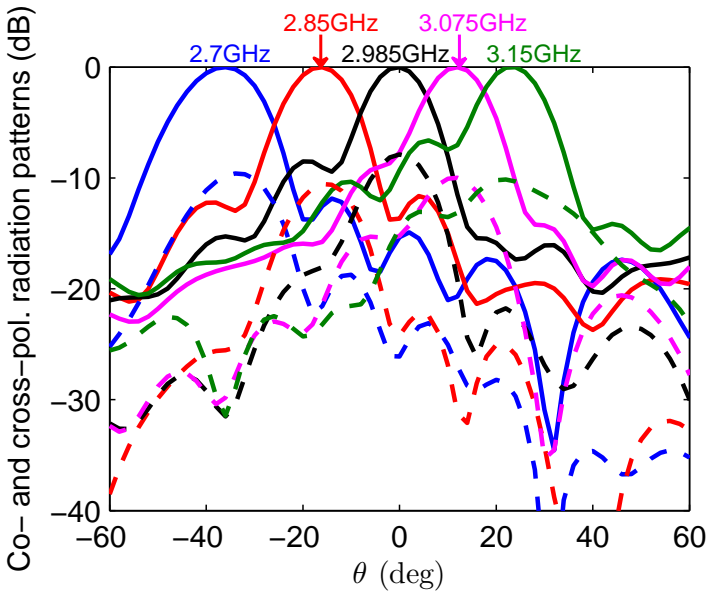

Fig. 28. Simulated radiation patterns of the optimized polarizationreconfigurable LWA at five frequencies within its LP bandwidth. Solid line: Co-polarization; Dash line: Cross-polarization.

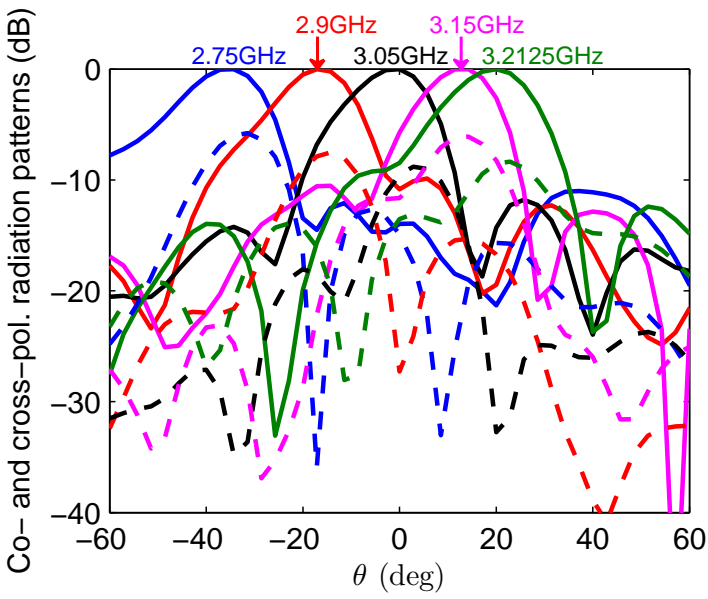

Fig. 29. Measured radiation patterns of the optimized polarizationreconfigurable LWA at five frequencies within its LP bandwidth. Solid line: Co-polarization; Dash line: Cross-polarization. 
TABLE II

COMPARISONS BETWEEN THE CP, LP AND POLARIZATION-RECONFIGURABLE LWAS DEVELOPED IN THIS PAPER

\begin{tabular}{cccccc}
\hline \hline Antenna & $\begin{array}{c}\text { frequency sweep } \\
\text { for beam scan }\end{array}$ & Scan range & Peak gain & Broadside gain & Gain variance \\
\hline Sim. CP LWA & $2.65-3.25 \mathrm{GHz}$ & $-34^{\circ}$ to $+32^{\circ}$ & $13.6 \mathrm{dBic}$ & $13.4 \mathrm{dBic}$ & $2.3 \mathrm{dBic}$ \\
\hline Mea. CP LWA & $2.75-3.35 \mathrm{GHz}$ & $-34.3^{\circ}$ to $+31.5^{\circ}$ & $13 \mathrm{dBic}$ & $12.3 \mathrm{dBic}$ & $3 \mathrm{dBic}$ \\
\hline Sim. LP LWA & $2.8-3.2 \mathrm{GHz}$ & $-32^{\circ}$ to $+26^{\circ}$ & $13.7 \mathrm{dBi}$ & $11.7 \mathrm{dBi}$ & $3.3 \mathrm{dBi}$ \\
\hline Mea. LP LWA & $2.85-3.275 \mathrm{GHz}$ & $-34.3^{\circ}$ to $+25.7^{\circ}$ & $13 \mathrm{dBi}$ & $10.6 \mathrm{dBi}$ & $3.45 \mathrm{dBi}$ \\
\hline $\begin{array}{c}\text { Sim. polarization-reconfigurable } \\
\text { LWA with CP state }\end{array}$ & $2.7-3.125 \mathrm{GHz}$ & $-30^{\circ}$ to $+16^{\circ}$ & $13.15 \mathrm{dBic}$ & $12.6 \mathrm{dBic}$ & $2.45 \mathrm{dBic}$ \\
\hline $\begin{array}{c}\text { Mea. polarization-reconfigurable } \\
\text { LWA with CP state }\end{array}$ & $2.75-3.2 \mathrm{GHz}$ & $-31.5^{\circ}$ to $+17.1^{\circ}$ & $12.8 \mathrm{dBic}$ & $11.8 \mathrm{dBic}$ & $3.3 \mathrm{dBic}$ \\
\hline $\begin{array}{c}\text { Sim. polarization-reconfigurable } \\
\text { LWA with LP state }\end{array}$ & $2.7-3.15 \mathrm{GHz}$ & $-36^{\circ}$ to $+24^{\circ}$ & $13 \mathrm{dBi}$ & $11.3 \mathrm{dBi}$ & $4 \mathrm{dBi}$ \\
\hline $\begin{array}{c}\text { Mea. polarization-reconfigurable } \\
\text { LWA with LP state }\end{array}$ & $2.75-3.2125 \mathrm{GHz}$ & $-34.3^{\circ}$ to $+20^{\circ}$ & $11.7 \mathrm{dBi}$ & $10.5 \mathrm{dBi}$ & $3.9 \mathrm{dBi}$ \\
\hline \hline
\end{tabular}

$+20^{\circ}$ at $2.75,2.9,3.05,3.15$, and $3.2125 \mathrm{GHz}$, respectively. The measured scanning range agrees well with the simulated one. The simulated and measured realized gain and cross polarization values are shown in Fig. 30 as functions of the source frequency. The measured realized gain varies between 7.8 (at $3.2125 \mathrm{GHz}$ ) and 11.7 (at $2.9 \mathrm{GHz}$ ) dBi across the -10-dB bandwidth from 2.75 to $3.2125 \mathrm{GHz}(462.5 \mathrm{MHz})$. The simulated realized gain varies between 9.0 (at $3.15 \mathrm{GHz}$ ) and 13.0 (at $2.85 \mathrm{GHz}$ ) dBi over the frequency band from 2.7 to $3.15 \mathrm{GHz}(450 \mathrm{MHz})$. The measure realized gain values are lower than the simulated one. This outcome is consistent with the fact that the measured radiation patterns shown in Fig. 29 have wider half-power beamwidths than their simulated ones. The measured cross polarization levels over the LP frequency band 2.75 to $3.2125 \mathrm{GHz}(463.5 \mathrm{MHz})$ range from -9.5 (at $3.05 \mathrm{GHz}$ ) to -6.0 (at $2.75 \mathrm{GHz}$ ) dB. The corresponding simulated values range from -11.0 (at $2.875 \mathrm{GHz}$ ) to -8.0 (at $3 \mathrm{GHz}$ ) dB over the frequency band from 2.7 to $3.15 \mathrm{GHz}$ (450 MHz). In comparison with their simulated values, the measured lower realized gain and higher cross polarization levels arise from inconsistent diode performance (all of the PIN diodes are switched ON for this LP state), as well as from fabrication and assembly errors. It is observed that the crosspolarization levels of the polarization-reconfigurable LWA in its LP state are higher than those of the fixed LP LWA. One could achieve similar cross-pol levels in its LP state, but at a cost of a deterioration in its CP performance. The cross-pol increase arises from the presence of the DC bias lines required to control the diode states. The simulated total efficiency of the polarization-reconfigurable LWA in its CP state is also shown in Fig. 26. It varies from $74 \%$ and $84 \%$ within the frequency band between 2.7 and $3.2 \mathrm{GHz}$.

\section{CONCLUSION}

A polarization-reconfigurable, beam scanning LWA was developed in this paper. It was demonstrated that scanning from the backward to the forward directions is continuous through broadside and that the antenna can be switched between its LP and CP states. This final optimized system was realized from two other reported antennas. Both a slot-loaded SIWbased CP LWA and a shorted-stub loaded modification of it to obtain a LP LWA were also designed, simulated, fabricated,

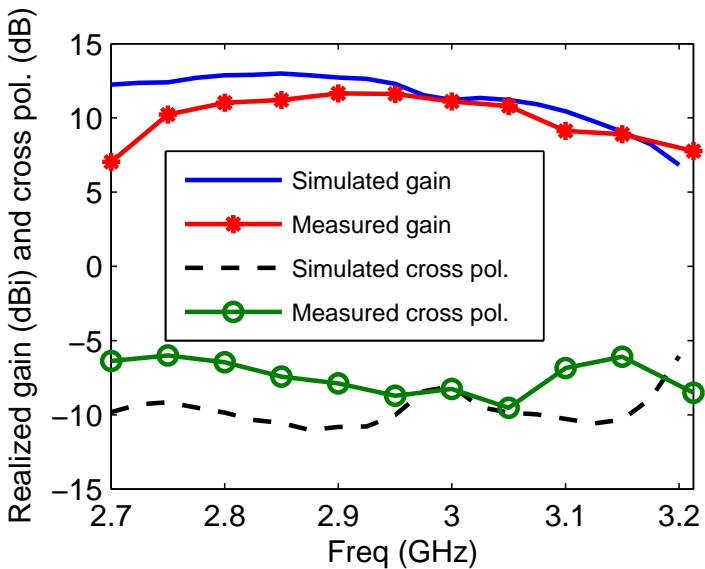

Fig. 30. Measured and simulated realized gain and cross polarization (cross pol.) values of the optimized polarization-reconfigurable LWA operating in its LP state as functions of the source frequency.

and measured. It was shown that the main beam of both of these non-reconfigurable antennas could also be scanned from the backward to the forward directions through broadside.

The prototypes of all three antennas verified their simulated performance characteristics. A complete comparison between the performance characteristics of these three antennas is given in Table II. The CP LWA beam was scanned continuously from $-28.6^{\circ}$ to $+31.5^{\circ}$ with a $3 \mathrm{~dB}$ gain variation. The LP LWA beam was scanned continuously from $-34.3^{\circ}$ to $+25.7^{\circ}$ with a $3.45 \mathrm{~dB}$ gain variation. The main beam of the polarizationreconfigurable LWA was scanned continuously from $-31.5^{\circ}$ to $+17.1^{\circ}$ with a $3.3 \mathrm{~dB}$ gain variation in its $\mathrm{CP}$ state and from $-34.3^{\circ}$ to $+20^{\circ}$ with a $3.9 \mathrm{~dB}$ gain variation in its LP state. The measured performance characteristics of all three antennas were shown to be in reasonable agreement with the simulated values. Note that the scanning range of the LWAs developed in this paper was determined by considering several performance characteristics. These include specific levels of their impedance matching, axial ratio, and gain variation values. The optimized reconfigurable LWA has a simple configuration, and its biasing circuits are easily realized. It is a highly promising antenna for future wireless communication systems requiring 
multi-functionality and high capacity.

\section{ACKNOWLEDGMENT}

The authors would like to thank Mr. Ming Li from Xiamen University for his assistance in the measurement campaign.

\section{REFERENCES}

[1] D. R. Jackson, C. Caloz, and T. Itoh, "Leaky-wave antennas," Proc. IEEE, vol. 100, no. 7, pp. 2194-2206, Jul. 2012.

[2] C. Jin and A. Alphones, "Leaky-wave radiation behavior from a double periodic composite right/left-handed substrate integrated waveguide," IEEE Trans. Antennas Propag., vol. 60, no. 4, pp. 1727-1735, Apr. 2012.

[3] Z. Li, J. H. Wang, M. E. Chen, and Z. Zhang, "New approach of radiation pattern control for leaky-wave antennas based on the effective radiation sections," IEEE Trans. Antennas Propag., vol. 63, no. 7, pp. 2867-2878, Jul. 2015.

[4] Z. Li, J. H. Wang, J. Duan, Z. Zhang, and M. E. Chen, "Analysis on the radiation property of the bounded modes of periodic leaky-wave structure with finite-length using a hybrid method," Sci. Rep., vol. 6, 22917, 2016.

[5] N. Nguyen-Trong, L. Hall, and C. Fumeaux, "Transmission-line model of nonuniform leaky-wave antennas," IEEE Trans. Antennas Propag., vol. 65, no. 3, pp. 883-893, Mar. 2016

[6] X.-X. Yang, L. Di, Y. Yu, and S. Gao, "Low-profile frequency-scanned antenna based on substrate integrated waveguide," IEEE Trans. Antennas Propag., vol. 65, no. 4, pp. 2051-2056, Apr. 2017.

[7] Y. J. Geng, J. H. Wang, Y. J. Li, Z. Li, M. E. Chen, and Z. Zhang, "Leaky-wave antenna array with a power-recycling feeding network for radiation efficiency improvement," IEEE Trans. Antennas Propag., vol. 65, no. 5, pp. 2689-2694, May 2017.

[8] N. Boskovic, B. Jokanovic, and M. Radovanovic, "Printed frequency scanning antenna arrays with enhanced frequency sensitivity and sidelobe suppression," IEEE Trans. Antennas Propag., vol. 65, no. 4, pp. 1757 1764, Apr. 2017

[9] A. J. Martinez-Ros, J. L. Gomez-Tornero, and G. Goussetis, "Planar leaky-wave antenna with flexible control of the complex propagation constant," IEEE Trans. Antennas Propag., vol. 60, no. 3, pp. 1625-1630, Mar. 2012.

[10] J. Liu, D. R. Jackson, and Y. Long, "Substrate integrated waveguide (SIW) leaky-wave antenna with transverse slots," IEEE Trans. Antennas Propag., vol. 60, no. 1, pp. 20-29, Jan. 2012.

[11] Y. Li, Q. Xue, E. K. N. Yung, and Y. Long, "The periodic half-width microstrip leaky-wave antenna with a backward to forward scanning capability," IEEE Trans. Antennas Propag., vol. 58, no. 3, pp. 963-966, Mar. 2010.

[12] D. K. Karmokar and K. P. Esselle, "Periodic U-slot-loaded dual-band half-width microstrip leaky-wave antennas for forward and backward beam scanning," IEEE Trans. Antennas Propag., vol. 63, no. 12, pp. 5372-5381, Dec. 2015.

[13] D. K. Karmokar, Y. J. Guo, P. Y. Qin, K. P. Esselle, and T. S. Bird, "Forward and backward beam scanning tri-band leaky-wave antenna," IEEE Antennas Wireless Propag. Lett., vol. 16, pp. 1891-1894, 2017.

[14] S. Paulotto, P. Baccarelli, F. Frezza, and D. R. Jackson, "A novel technique for open-stopband suppression in 1-D periodic printed leakywave antennas," IEEE Trans. Antennas Propag., vol. 57, no. 7, pp. 18941906, Jul. 2009.

[15] D. Comite et al., "A dual-layer planar leaky-wave antenna designed for linear scanning through broadside," IEEE Antennas Wireless Propag. Lett., vol. 16, pp. 1106-1110, 2017.

[16] J. Liu, W. Zhou, and Y. Long, "A simple technique for open-stopband suppression in periodic leaky-wave antennas using two nonidentical elements per unit cell," IEEE Trans. Antennas Propag., vol. 66, no. 6, pp. 2741-2751, Jun. 2018.

[17] J. T. Williams, P. Baccarelli, S. Paulotto, and D. R. Jackson, "1D combline leaky-wave antenna with the open-stopband suppressed: Design considerations and comparisons with measurements," IEEE Trans. Antennas Propag., vol. 61, no. 9, pp. 4484-4492, Jun. 2013.

[18] Y.-L. Lyu, X. X. Liu, P. Y. Wang, D. Erni, Q. Wu, C. Wang, N. Y. Kim, and F.-Y. Meng, "Leaky-wave antennas based on noncutoff substrate integrated waveguide supporting beam scanning from backward to forward," IEEE Trans. Antennas Propag., vol. 64, no. 6, pp. 2155-2164, Jun. 2016.

[19] A. Kianinejad, Z. N. Chen, and C. W. Qiu, "A single-layered spoof plasmon leaky wave antenna with consistent gain," IEEE Trans. Antennas Propag., vol. 65, no. 2, pp. 681-687, Feb. 2017
[20] C. S. Prasad and A. Biswas, "Dielectric image line-based leaky-wave antenna for wide range of beam scanning through broadside," IEEE Trans. Antennas Propag., vol. 65, no. 8, pp. 4311-4315, Aug. 2017.

[21] A. Lai, T. Itoh, and C. Caloz, "Composite right/left-handed transmission line metamaterials," IEEE Microw. Mag., vol. 5, no. 3, pp. 34-50, Sep. 2004.

[22] S. Paulotto, P. Baccarelli, F. Frezza, and D. R. Jackson, "Full-wave modal dispersion analysis and broadside optimization for a class of microstrip CRLH leaky-wave antennas," IEEE Trans. Microw. Theory Techn., vol. 56, no. 12, pp. 2826-2837, Dec. 2008.

[23] Y. Dong and T. Itoh, "Composite right/left-handed substrate integrated waveguide and half mode substrate integrated waveguide leaky-wave structures," IEEE Trans. Antennas Propag., vol. 59, no. 3, pp. 767-775, Mar. 2011.

[24] Nasimuddin, Z. N. Chen, and X. Qing, "Multilayered composite right/left-handed leaky-wave antenna with consistent gain," IEEE Trans. Antennas Propag., vol. 60, no. 11, pp. 5056-5062, Nov. 2012.

[25] Nasimuddin, Z. N. Chen, and X. Qing, "Substrate integrated metamaterial-based leaky-wave antenna with improved boresight radiation bandwidth," IEEE Trans. Antennas Propag., vol. 61, no. 7, pp. $3451-$ 3457, Jul. 2013.

[26] W. Cao, Z. N. Chen, W. Hong, B. Zhang, and A. Liu, "A beam scanning leaky-wave slot antenna with enhanced scanning angle range and flat gain characteristic using composite phase-shifting transmission line," IEEE Trans. Antennas Propag., vol. 62, no. 11, pp. 5871-5875, Nov. 2014.

[27] G. C. Wu, G. M. Wang, H. X. Peng, X. J. Gao, and J. G. Liang, "Design of leaky-wave antenna with wide beam-scanning angle and low cross-polarisation using novel miniaturised composite right/left-handed transmission line," IET Microw. Antennas Propag.,, vol. 10, no. 7, pp. 777-783, May 2016.

[28] D.-F. Guan, P. You, Q. Zhang, Z.-H. Lu, S.-W. Yong, and K. Xiao, "A wide-angle and circularly polarized beam-scanning antenna based on microstrip spoof surface plasmon polariton transmission line," IEEE Antennas Wireless Propag. Lett., vol. 16, pp. 2538-2541, 2017.

[29] Y.-L. Lyu, F.-Y. Meng, G.-H. Yang, D. Erni, Q. Wu, and K. Wu, "Periodic SIW leaky-wave antenna with large circularly-polarized beam scanning range," IEEE Antennas Wireless Propag. Lett., vol. 16, pp. $2493-$ 2496, 2017.

[30] P.-Y. Qin, A. R. Weily, Y. J. Guo, and C.-H. Liang, "Polarization reconfigurable U-slot patch antenna," IEEE Trans. Antennas Propag., vol. 58, no. 10 , pp. 3383-3388, Oct. 2010.

[31] W. Lin and H. Wong, "Polarization reconfigurable wheel-shaped antenna with conical-beam radiation pattern," IEEE Trans. Antennas Propag., vol 63, no. 2, pp. 491-499, Feb. 2015.

[32] W. Lin and H. Wong, "Wideband circular polarization reconfigurable antenna," IEEE Trans. Antennas Propag., vol. 63, no. 12, pp. 5938-5944, Dec. 2015.

[33] S.-L. Chen, F. Wei, P.-Y. Qin, Y. J. Guo, and X. Chen, "A multi-linear polarization reconfigurable unidirectional patch antenna," IEEE Trans. Antennas Propag., vol. 65, no. 8, pp. 4299-4304, Aug. 2017.

[34] Z. Chen and H. Wong, "Liquid dielectric resonator antenna with circular polarization reconfigurability," IEEE Trans. Antennas Propag., vol. 66, no. 1, pp. 444-449, Jan. 2018.

[35] Y. J. Guo, P.-Y. Qin, S.-L. Chen, W. Lin, and R. W. Ziolkowski, "Advances in reconfigurable antenna systems facilitated by innovative technologies,” IEEE Access, vol. 6, pp. 5780-5794, Jan. 2018.

[36] M. R. M. Hashemi and T. Itoh, "Composite right/left-handed leaky-wave transmission-line with switchable polarization capability," in Proc. 41th. Eur. Microw. Conf., Oct. 2011, pp. 495-498.

[37] Y. Dong and T. Itoh, "Substrate integrated composite right-/left-handed leaky-wave structure for polarization-flexible antenna application," IEEE Trans. Antennas Propag., vol. 60, no. 2, pp. 760-771, Feb. 2012

[38] ANSYS HFSS-High Frequency Electromagnetic Field Simulation. [Online]. Available: http://www.ansys.com/Products/Electronics/ANSYSHFSS.

[39] D. K. Karmokar, Y. J. Guo, P.-Y. Qin, S.-L. Chen, and T. S. Bird, "Substrate integrated waveguide-based periodic backward-to-forward scanning leaky-wave antenna with low cross-polarization," IEEE Trans. Antennas Propag., vol. 66, no. 8, pp. 3846-3856, Aug. 2018.

[40] C. A. Balanis, "Antenna theory: A review," Proc. IEEE, vol. 80, no. 1, pp. 7-23, Jan. 1992.

[41] T. Oh, Y.-G. Lim, C.-B. Chae, and Y. Lee, "Dual-polarization slot antenna with high cross-polarization discrimination for indoor small-cell MIMO systems," IEEE Antennas Wireless Propag. Lett., vol. 14, pp. 374377, 2014.

[42] H.-R. Chuang and L.-C. Kuo, "3-D FDTD design analysis of a 2.4-GHz polarization-diversity printed dipole antenna with integrated balun and 
polarization-switching circuit for WLAN and wireless communication applications," IEEE Trans. Microw. Theory Techn., vol. 51, no. 2, pp. 374-381, Feb. 2003.

[43] L. Y. Ji, P. Y. Qin, Y. J. Guo, C. Ding, and S. X. Gong, "A wideband polarization reconfigurable antenna with partially reflective surface," IEEE Trans. Antennas Propag., vol. 64, no. 10, pp. 4534-4538, Oct. 2016.

[44] S.-L. Chen, P.-Y. Qin, C. Ding, and Y. J. Guo, "Cavity-backed proximitycoupled reconfigurable microstrip antenna with agile polarizations and steerable beams," IEEE Trans. Antennas Propag., vol. 65, no. 10, pp. 5553-5558, Oct. 2017

[45] Datasheet Bar50-02L PIN Diodes. (2017, Oct. 2). Infineon Technologies, Application Note [Online]. Available: http://www.infineon.com.

[46] Chip Inductors-0402HP Series (1005), Coilcraft Corporation [Online]. Available: https://www.coilcraft.com/0402hp.cfm

[47] Chip Inductors-0402HP Series (1005), Coilcraft Corporation [Online]. Available: https://www.coilcraft.com/pdfs/spice 0402hp.pdf

[48] R. B. V. B. Simorangkir, Y. Yang, K. P. Esselle, and B. A. Zeb, "A method to realize robust flexible electronically tunable antennas using polymer-embedded conductive fabric," IEEE Trans. Antennas Propag., vol. 66 , no. 1 , pp. 50-58, Jan. 2018.

[49] Y. Li and A. Abbosh, "Reconfigurable reflectarray antenna using singlelayer radiator controlled by PIN diodes," IET Microw. Antennas Propag., vol. 9, no. 7, pp. 664-671, May 2015.

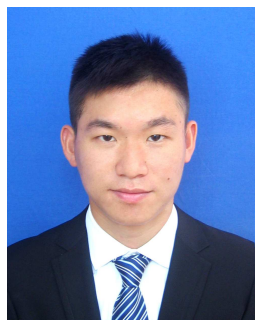

Shu-Lin Chen (S'16) was born in Hubei Province, China. He received the B.S. degree in electrical engineering from Fuzhou University, China, in 2012, and the M.S. degree in electromagnetic field and microwave technology from Xiamen University, China, in 2015. Since 2016, he has been working towards the $\mathrm{PhD}$ degree in Engineering with the Global Big Data Technologies Centre (GBDTC), University of Technology Sydney (UTS), Australia.

His research interests include reconfigurable antennas, leaky-wave antennas, millimeter wave antennas, and adaptive array processing. He was a finalist of ISAP 2017 best paper competition, and his paper was listed as an Honorary Mention in APS-URSI 2017.

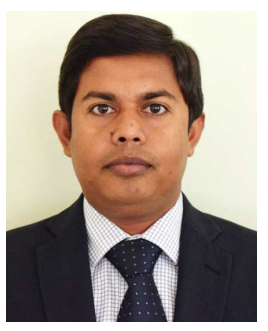

Debabrata K. Karmokar (S'12-M'15) received the B.Sc. degree in electrical \& electronic engineering (EEE) from the Khulna University of Engineering \& Technology (KUET), Khulna, Bangladesh, in 2007 and the PhD degree in Electronic Engineering from Macquarie University, Sydney, NSW, Australia in 2015. He was an Assistant Professor and a member of CRTS in the department of EEE, KUET, and an Assistant Director of Students' Welfare of the University. Between 2012 and 2015, he was a Research Assistant, a Casual Academic, and also a Secretary of the IEEE Student Branch, at Macquarie University. From 2016 to 2019 he was a Postdoctoral Research Associate with the Global Big Data Technologies Centre (GBDTC), University of Technology Sydney (UTS), Australia. He is currently a Lecturer with the School of Engineering, Macquarie University, Australia.

Dr. Karmokar is a recipient of several scholarships and awards including a District Council Scholarship from Satkhira District Council, Bangladesh, Technical Scholarship from KUET, a Commonwealth-funded International Postgraduate Research Scholarship (IPRS) together with an International Macquarie University Research Excellence Scholarship (iMQRES), an OCE $\mathrm{PhD}$ Scholarship from the Commonwealth Scientific and Industrial Research Organisation (CSIRO) ICT Centre, Marsfield, Australia, the First Prize in the Poster Competition at the Engineering Symposium 2015, Macquarie University, Young Scientist Award from Venus International Foundation in 2018, and URSI Young Scientist Award in 2019. He is serving as a reviewer for several journals of IEEE, IET, Wiley, Springer and Elsevier.

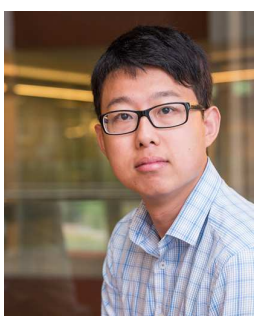

Pei-Yuan Qin (M'13) was born in Liaoning Province, China, in 1983. He received a Bachelor Degree in Electronic Engineering from Xidian University, Xi'an, China, in 2006, and a joint Ph.D. Degree from Xidian University and Macquarie University, Australia, in electromagnetic fields and microwave technology in 2012.

From 2012 to 2015, he was a Postdoctoral Research Fellow in Commonwealth Scientific and Industrial Research Organisation (CSIRO), Australia. From 2015 to 2016, he was a Chancellor's Postdoctoral Research Fellow/Lecturer with University of Technology Sydney (UTS), Australia. Since 2017, he is a Senior Lecturer with UTS. His research interests are in the areas of reconfigurable antennas, phase shifters, reconfigurable reflectarrays, and MIMO communications.

Dr. Qin was a recipient of an Australia Research Council Discovery Early Career Researcher Award and also a recipient of the International Macquarie University Research Excellence Scholarship. He was awarded the Vice Chancellor's Commendation for academic excellence by Macquarie University. Since 2017, he has been serving as an Associate Editor of the IEEE Antennas and Wireless Propagation Letters.

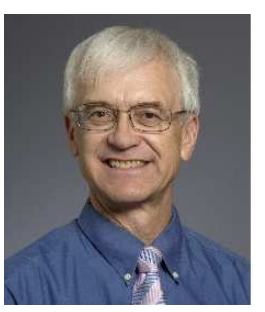

Richard W. Ziolkowski (M'87-SM'91-F'94) received the B.Sc. (magna cum laude) degree (Hons.) in physics from Brown University, Providence, RI, USA, in 1974; the M.S. and Ph.D. degrees in physics from the University of Illinois at UrbanaChampaign, Urbana, IL, USA, in 1975 and 1980, respectively; and the Honorary Doctorate degree from the Technical University of Denmark, Kongens Lyngby, Denmark in 2012.

$\mathrm{He}$ is currently a Distinguished Professor in the Global Big Data Technologies Centre in the Faculty of Engineering and Information Technologies (FEIT) at the University of Technology Sydney, Ultimo NSW, Australia. He became a Professor Emeritus at the University of Arizona in 2018, where he was a Litton Industries John M. Leonis Distinguished Professor in the Department of Electrical and Computer Engineering in the College of Engineering and was also a Professor in the College of Optical Sciences. He was the Computational Electronics and Electromagnetics Thrust Area Leader with the Lawrence Livermore National Laboratory, Engineering Research Division, in Livermore, CA before joining The University of Arizona, Tucson, AZ, USA, in 1990.

Prof. Ziolkowski is the recipient of the 2019 IEEE Electromagnetics Award (IEEE Field Award). He is a Fellow of the Optical Society of America (OSA, 2006), and of the American Physical Society (APS, 2016). He was the Australian DSTO Fulbright Distinguished Chair in Advanced Science and Technology from 2014-2015. He was a 2014 Thomas-Reuters Highly Cited Researcher. He served as the President of the IEEE Antennas and Propagation Society in 2005. He is also actively involved with the URSI, OSA and SPIE professional societies.

His current research interests include the application of new mathematical and numerical methods to linear and nonlinear problems dealing with the interaction of electromagnetic and acoustic waves with complex linear and nonlinear media, as well as metamaterials, metamaterial-inspired structures, nano-structures, and other classical and quantum applications-specific configurations. 


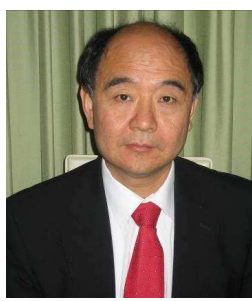

Y. Jay Guo (Fellow'2014) received a Bachelor Degree and a Master Degree from Xidian University in 1982 and 1984, respectively, and a PhD Degree from Xian Jiaotong University in 1987, all in China. His research interest includes antennas, $\mathrm{mm}$-wave and $\mathrm{THz}$ communications and sensing systems as well as big data technologies. He has published over 400 research papers and holds 24 patents in antennas and wireless systems. He is a Fellow of the Australian Academy of Engineering and Technology, a Fellow of IEEE and a Fellow of IET, and a member of the College of Experts of Australian Research Council (ARC). He has won a number of most prestigious Australian national awards, and was named one of the most influential engineers in Australia in 2014 and 2015.

Prof. Guo is a Distinguished Professor and the founding Director of Global Big Data Technologies Centre at the University of Technology Sydney (UTS),
Australia. Prior to this appointment in 2014, he served as a Director in CSIRO for over nine years, directing a number of ICT research portfolios. Before joining CSIRO, he held various senior technology leadership positions in Fujitsu, Siemens and NEC in the U.K.

Prof. Guo has chaired numerous international conferences. $\mathrm{He}$ is the Chair Elect of International Steering Committee, International Symposium on Antennas and Propagation (ISAP). He was the International Advisory Committee Chair of IEEE VTC2017, General Chair of ISAP2015, iWAT2014 and WPMC'2014, and TPC Chair of 2010 IEEE WCNC, and 2012 and 2007 IEEE ISCIT. He served as Guest Editor of special issues on "Antennas for Satellite Communications" and "Antennas and Propagation Aspects of 6090GHz Wireless Communications," both in IEEE Transactions on Antennas and Propagation, Special Issue on "Communications Challenges and Dynamics for Unmanned Autonomous Vehicles," IEEE Journal on Selected Areas in Communications (JSAC), and Special Issue on "5G for Mission Critical Machine Communications", IEEE Network Magazine. 